\title{
Endothelial NOS, estrogen receptor $\beta$, and HIFs cooperate in the activation of a prognostic transcriptional pattern in aggressive human prostate cancer
}

\author{
Simona Nanni,1,2,3 Valentina Benvenuti,1,2 Annalisa Grasselli,1,2 Carmen Priolo,2,4 \\ Aurora Aiello, ${ }^{1,3}$ Stefania Mattiussi, ${ }^{5}$ Claudia Colussi, ${ }^{6}$ Vittoria Lirangi, ${ }^{1}$ Barbara Illi, ${ }^{6}$ \\ Manuela D'Eletto, ${ }^{1}$ Anna Maria Cianciulli, 7 Michele Gallucci, ${ }^{8}$ Piero De Carli, ${ }^{8}$ Steno Sentinelli, ${ }^{9}$ \\ Marcella Mottolese, ${ }^{9}$ Paolo Carlini, ${ }^{10}$ Lidia Strigari, ${ }^{11}$ Stephen Finn, ${ }^{4}$ Elke Mueller, ${ }^{4}$ \\ Giorgio Arcangeli, ${ }^{12}$ Carlo Gaetano, ${ }^{5}$ Maurizio C. Capogrossi, ${ }^{5}$ Raffaele Perrone Donnorso, ${ }^{9}$ \\ Silvia Bacchetti, ${ }^{1}$ Ada Sacchi, ${ }^{1}$ Alfredo Pontecorvi, ${ }^{2,13}$ Massimo Loda, ${ }^{4}$ and Antonella Farsetti ${ }^{13,13}$ \\ ${ }^{1}$ Department of Experimental Oncology, Regina Elena Cancer Institute, Rome, Italy. ${ }^{2}$ Department of Endocrinology, \\ Catholic University, Rome, Italy. ${ }^{3}$ Institute of Neurobiology and Molecular Medicine, National Research Council (CNR), Rome, Italy. \\ 4Department of Medical Oncology, Dana-Farber Cancer Institute, and Department of Pathology, Brigham and Women's Hospital, \\ Harvard Medical School, Boston, Massachusetts, USA. 5Laboratorio di Patologia Vascolare, Istituto Dermopatico dell'Immacolata (IDI), \\ Rome, Italy. ${ }^{6}$ Centro Cardiologico Monzino, Milan, Italy. ${ }^{7}$ Department of Clinical Pathology, ${ }^{8}$ Department of Urology, \\ 9Department of Pathology, ${ }^{10}$ Department of Medical Oncology, ${ }^{11}$ Laboratory of Medical Physics and Expert Systems, \\ ${ }^{12}$ Department of Radiotherapy, and ${ }^{13}$ Rome Oncogenomic Center, Regina Elena Cancer Institute, Rome, Italy.
}

\begin{abstract}
The identification of biomarkers that distinguish between aggressive and indolent forms of prostate cancer (PCa) is crucial for diagnosis and treatment. In this study, we used cultured cells derived from prostate tissue from patients with PCa to define a molecular mechanism underlying the most aggressive form of PCa that involves the functional activation of eNOS and HIFs in association with estrogen receptor $\beta(E R \beta)$. Cells from patients with poor prognosis exhibited a constitutively hypoxic phenotype and increased NO production. Upon estrogen treatment, formation of ER $\beta / \mathrm{eNOS}$, ER $\beta / \mathrm{HIF}-1 \alpha$, or ER $\beta / \mathrm{HIF}-2 \alpha$ combinatorial complexes led to chromatin remodeling and transcriptional induction of prognostic genes. Tissue microarray analysis, using an independent cohort of patients, established a hierarchical predictive power for these proteins, with expression of eNOS plus ER $\beta$ and nuclear eNOS plus HIF- $2 \alpha$ being the most relevant indicators of adverse clinical outcome. Genetic or pharmacologic modulation of eNOS expression and activity resulted in reciprocal conversion of the transcriptional signature in cells from patients with bad or good outcome, respectively, highlighting the relevance of eNOS in PCa progression. Our work has considerable clinical relevance, since it may enable the earlier diagnosis of aggressive PCa through routine biopsy assessment of eNOS, ER $\beta$, and HIF-2 $\alpha$ expression. Furthermore, proposing eNOS as a therapeutic target fosters innovative therapies for PCa with NO inhibitors, which are employed in preclinical trials in non-oncological diseases.
\end{abstract}

\section{Introduction}

In the clinical management of prostate cancer $(\mathrm{PCa})$, the second most common neoplasia in men worldwide (1), the ability to distinguish between aggressive and indolent forms of the disease is critical. Thus, therapeutic approaches would be substantially improved by the identification of the molecular mechanisms involved in tumor progression and the key biomarkers capable of improving patients' stratification at diagnosis, by discriminating between those at risk for relapse and those with indolent tumors not requiring further intervention beyond surgery.

Authorship note: Simona Nanni, Valentina Benvenuti, and Annalisa Grasselli contributed equally to this work.

Conflict of interest: Massimo Loda is a consultant for Novartis and Director of the Center for Molecular Oncologic Pathology, Dana-Farber Cancer Institute.

Nonstandard abbreviations used: AR, androgen receptor; DSS, disease-specific survival; E2, 17 $\beta$-estradiol; ER, estrogen receptor; ERE, estrogen-responsive element; HRE, hypoxia-responsive element; hTERT, human telomerase catalytic subunit; PCa, prostate cancer; PSA, prostate-specific antigen; TMA, tissue microarray.

Citation for this article: J. Clin. Invest. 119:1093-1108 (2009). doi:10.1172/JCI35079.
Recently, we and others $(2,3)$ reported on the induction of genes involved in the cell response to hypoxia in prostate, breast, and ovarian cancers and on the relevance of this phenomenon as predictor of adverse clinical outcome, suggesting that HIFs, beside their well-established role in the biology of solid tumors, represent key transcription factors specifically in endocrine tumors. High expression of the hypoxia response signature in breast cancers has a predictive power greater than parameters such as response to chemotherapy, estrogen receptors (ERs), tumor size and grade, angiogenic invasion, or age (3). In particular, HIF- $1 \alpha$ appears to promote early invasive lesions (4) and, indeed, in PCa is expressed at early stages $(5,6)$, supporting its specific role as predictor of poor prognosis. The more aggressive prostate tumors, in fact, are characterized by increased expression of HIF- $1 \alpha$, HIF- $2 \alpha$, and HIF- $1 \beta$ and their gene targets. Moreover, cells from these tumors exhibit a constitutive "hypoxic" phenotype, even in normoxic conditions (see below), suggesting that hypoxia may confer a significant growth advantage (7), thus promoting and shaping cancer evolution $(4,8)$. 
Another key molecule, on which a number of studies on PCa etiopathogenesis have been focused in the last years, is the ER. Although androgens have been traditionally considered the major hormonal regulators of the prostate gland, increasing experimental evidence has recently attributed an equally important function to estrogens (9). The first ER expressed in the fetal prostate, and the predominant form in its epithelium is $\mathrm{ER} \beta$, which, together with the androgen receptor (AR), appears to mediate the initial stages of gland development $(10,11)$. Discrepancies in the literature make it difficult to define the precise biological role of the 2 ER subtypes, ER $\beta$ and $\mathrm{ER} \alpha$, in PCa $(9,12,13)$; however, the main function of ER $\beta$ appears to be associated with cell survival (14). Specifically, the retained expression of ER $\beta$ in the percentage of recurrent $\mathrm{PCa}$ associated with increased mortality (15) and in all metastatic lesions (16) is strongly suggestive of a critical involvement of this receptor in PCa progression.

Along the hypoxia and ER pathways lays the eNOS, whose expression, although abundant in endothelial cells, is widely distributed among different tissue and cell types and in tumors, including PCa (see below). The eNOS gene promoter harbors hypoxia and ER response elements, and, in fact, eNOS activity is regulated by hypoxia and/or estrogen (17-20). In addition, the product of eNOS, NO, affects HIF- $1 \alpha$ synthesis and accumulation in normoxia, indicating the existence of a regulatory loop between these molecules (21-24).

Finally, eNOS and $\mathrm{NO}$ also play an important role in tumorigenesis and tumor maintenance (25-27). Remarkably, in human endothelial cells, eNOS and ER form a nuclear complex that regulates transcription of the human telomerase catalytic subunit (hTERT) (18), a molecule that is an early marker of PCa development $(28,29)$.

Since $h T E R T$ and several other genes belonging to a prognostic transcriptional signature (2) respond to both estrogens or hypoxia, we queried whether ERs, HIFs and eNOS may cooperate in PCa by co-regulating their transcription. To investigate this potential mechanism in vitro, we have used our ex vivo experimental model, consisting of immortalized cell populations derived from a cohort of patients with $\mathrm{PCa}$, characterized by a prognostic gene profile, discriminating cells from tumors with poor versus good prognosis (G1 versus G2) (2). We have then validated our findings in vivo on tissue microarrays (TMAs) of primary tumors from an independent cohort of patients with known clinical outcome. Through these approaches, we have been able to demonstrate the existence of a functional cooperation among the estrogen-, hypoxia-, and NO-dependent signaling pathways in the activation of a transcriptional program specifically associated with aggressive PCa.

\section{Results}

Generation and characterization of immortalized prostate tumor cells. On the basis of a defined transcriptional signature with prognostic relevance in $\mathrm{PCa}(2)$, we set out to gather interrelated information aimed at identifying molecular targets and mechanisms potentially relevant to $\mathrm{PCa}$ progression and at determining the expression pattern of genes essential to individual patient prognosis. To this end, we previously derived epithelial cell strains from prostate tissue freshly explanted from patients with benign prostate hyperplasia or clinically localized carcinoma (2). These cells, even when from tumor tissue, had a limited lifespan in culture (at most 25 population doublings; Figure 1A). Telomerase was expressed at variable levels in these populations (2) but waned over time (data not shown). Telomerase reconstitution by transduction of $b T E R T$ did not affect lifespan, and transduction of SV40 large T antigen extended lifespan to about 60 population doublings (data not shown). Cotransduction of $h T E R T$ and $\mathrm{T}$ antigen, however, resulted in unlimited proliferation (immortalization) of nearly all cell strains (25 of 29; Figure 1A). Immortal populations retained the markers of the epithelial luminal phenotype of parental cells (2), as assessed by immunocytochemistry (see Supplemental Methods), being positive for AR, cytokeratin 8, and prostate-specific antigen (PSA) but negative for high-molecular-weight cytokeratin, $\mathrm{p} 63$, vimentin, and $\alpha$-smooth muscle actin (Figure 1B and data not shown). All cells expressed ER $\beta$ but not ER $\alpha$ (Figure 1, B and C) and showed functional responsiveness of AR to dihydrotestosterone (data not shown).

Maintenance of a prognostic transcriptional signature upon cell immortalization. All immortalized cell lines maintained the mRNA expression pattern seen in parental cells for genes of the transcriptional signature discriminating between poor (G1 group) and good (G2 group) prognosis (2). Expression of the hypoxia response family genes, their targets (30), and other genes, such as the classical estrogen target PS2 or AKT (assessed by quantitative RT-PCR; see Supplemental Methods), was significantly different between G1 and G2 cells $(P=0.028$ and $P=0.007$, respectively; Figure 1D and data not shown). Protein levels were determined by Western blot in C27IM and C38IM cells, representing the G1 and G2 groups, respectively. Expression of HIF-1 $\alpha$ and HIF- $2 \alpha$ (Figure 1E) was detected already in normoxia in C27IM cells, indicating that they have a constitutively hypoxic phenotype. In C38IM cells, on the other hand, HIF- $1 \alpha$ appeared to be absent and HIF- $2 \alpha$ was barely detectable. Finally, hypoxia enhanced, albeit slightly, both HIF-1 $\alpha$ and HIF- $2 \alpha$ in C27IM cells, while in C38IM cells, it strongly enhanced HIF- $1 \alpha$ and had no apparent effect on HIF- $2 \alpha$. A more in depth analysis of this response is presented below. The substantial difference in ARNT mRNA could be confirmed at the protein level only in a subset of the cell lines (Figure 1E).

Expression of eNOS, a target of HIFs and estrogens, was examined by confocal microscopy (see Supplemental Methods) in cells of both groups. Expression of the protein (Figure 2A), in terms of both amount and number of positive cells, was significantly higher in G1 than G2 cells $(P<0.005)$. Most interestingly, in both cell types the protein was localized almost exclusively in the nucleus even, in the absence of the hypoxic stimulus. Nuclear relocalization of eNOS was observed in our recent study on endothelial cells upon exposure to estrogen (18). Thus, eNOS translocation to the nucleus in PCa cells may be dependent upon constitutive deregulation of hormone signaling in this endocrine tumor. In agreement with their higher levels of eNOS expression, G1 cells produced significantly higher amounts of $\mathrm{NO}$ than either benign prostate hyperplasia or $\mathrm{G} 2$ cells (Figure 2B), indicating that downstream effectors of the estrogen and hypoxia-inducible pathways are constitutively active only in G1 cells and even under normoxia.

Responsiveness to bypoxia of G1 and G2 cells. To more carefully characterize the constitutive hypoxic phenotype of $\mathrm{G} 1$ cells and evaluate the integrity of the hypoxia response in both G1 and G2 cells, we analyzed, by confocal microscopy, the expression of both HIF- $1 \alpha$ and HIF- $2 \alpha$ and quantified their nuclear/cytoplasmic distribution in normoxia or hypoxia in 4 cell lines belonging to both groups. As shown in Figures 3 and 4, already in normoxia, G1 cells (C27IM) had significant expression of HIF- $1 \alpha$ in the cytoplasm, together with a high percentage of nuclei positive for both HIF- $1 \alpha$ and HIF- $2 \alpha$. Expression of both proteins in G2 cells (C39IM), whether in the cytoplasm or the nucleus, was significantly lower. We attribute the apparent discrepancy between the lack of signal for HIF- $1 \alpha / \mathrm{HIF}-2 \alpha$ 
A

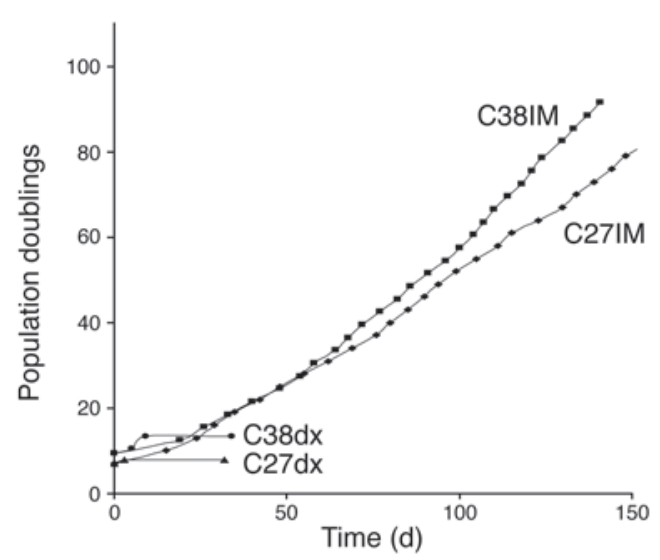

B

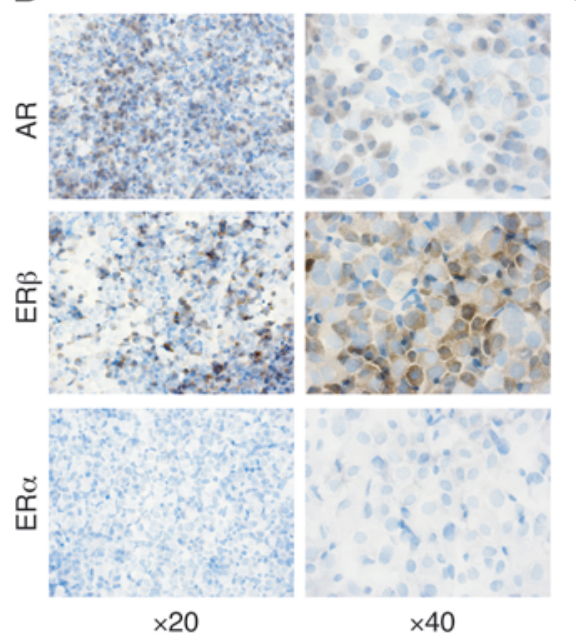

C

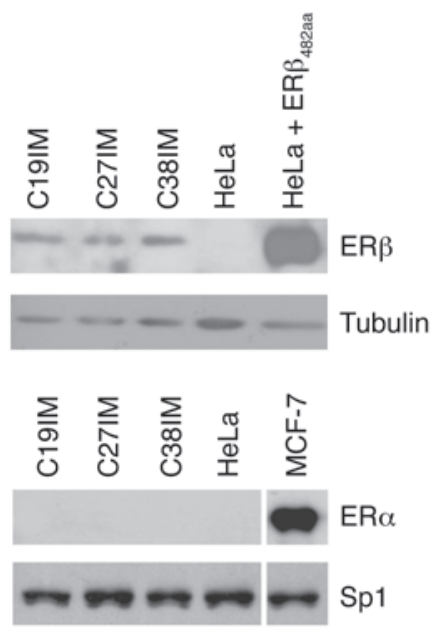

D
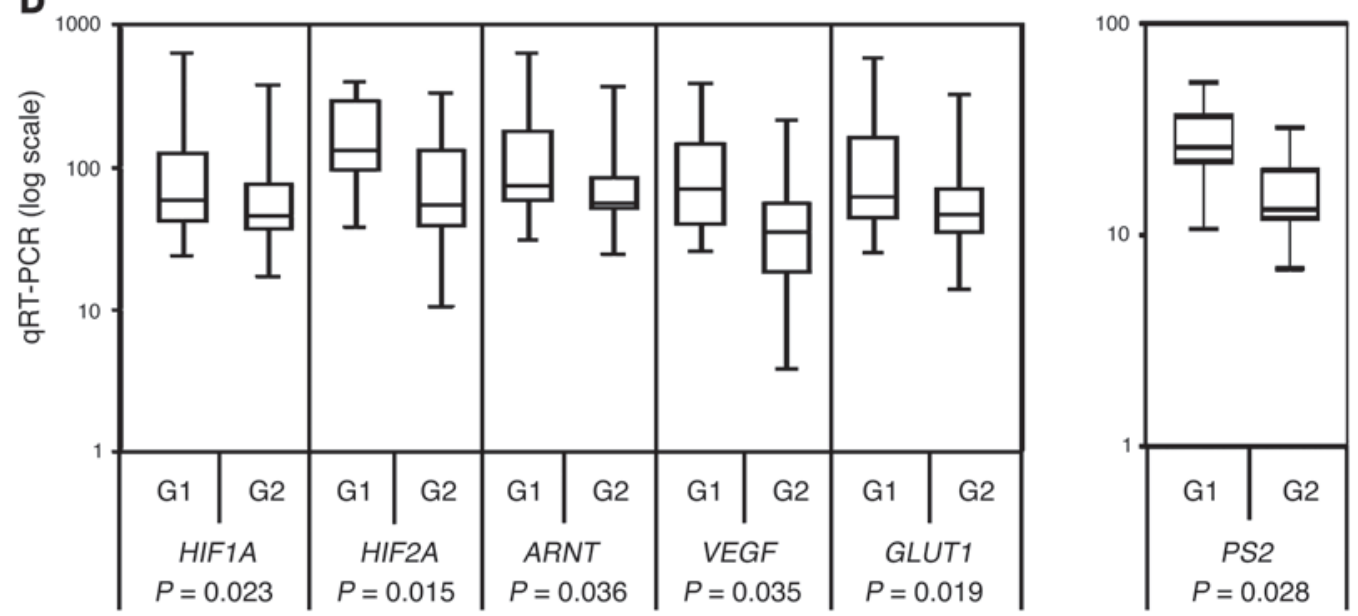

$\mathbf{E}$
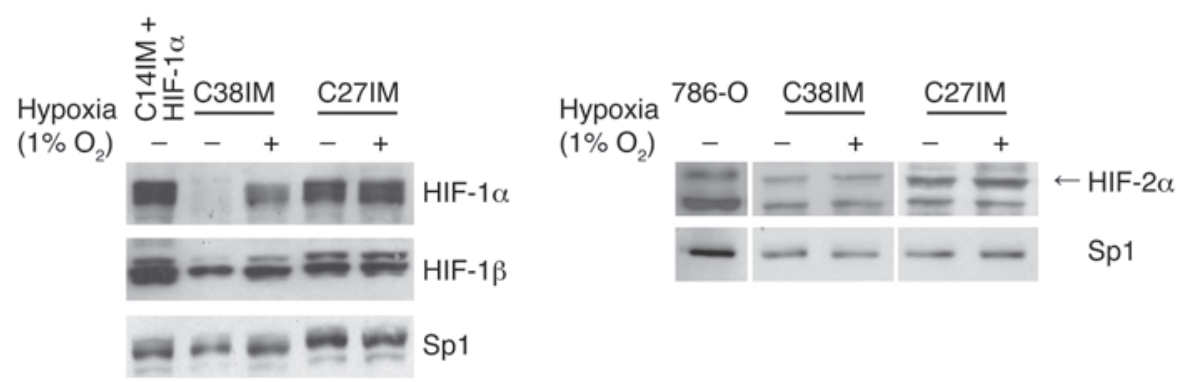

Figure 1

Generation, characterization, and maintenance of the prognostic signature in immortalized human prostate epithelial cell lines. (A) Growth (population doublings) of cell populations derived from tumoral prostate before (C27 and C38) and after retrovirus-mediated transduction of the hTERT and SV40 large T antigen genes (C27IM and C38IM, respectively). Cells were infected at early passage and cultured as described in Supplemental Methods. (B) Steroid receptor expression (AR) by immunocytochemistry. Original magnification is $\times 20$ or $\times 40$ as indicated. (C) ER $\beta$ and ER $\alpha$ detected by Western Blot in total or nuclear extracts, respectively, of PCa-derived cells (C19IM, C27IM, and C38IM). HeLa cells were negative control. HeLa cells transiently transfected with ER $\beta$ (HeLa + ER $\beta_{485 a a}$ ) and MCF-7 cells were positive control. Tubulin and nuclear protein Sp1 were loading control. (D) Analysis by quantitative RT-PCR (qRT-PCR) of genes from the prognostic expression signature in immortalized PCa cells (PCa-IM) $(n=25)$ from the poor (G1) or good (G2) prognosis groups. Data are presented as box plot and whisker (see Supplemental Methods). Boxes show medians and upper and lower quartiles and whiskers indicate minimum and maximum values. (E) Western blot of HIF-1 $\alpha$, HIF-2 $\alpha$, and HIF-1 $\beta$ in PCa-IM cells from the G1 (C27IM) or G2 (C38IM) groups in normoxia or hypoxia ( $1 \% \mathrm{O}_{2}$ for 3 hours). PCa cells transfected with an HIF-1 $\alpha$-expression vector (C14IM + HIF-1 $\alpha)$ and von Hippel-Lindau-deficient carcinoma 786-O cells were positive controls. Sp1 was the loading control. White lines indicate extracts that were run in noncontiguous lanes. 
research article

A

G1

- C27IM

C11IM
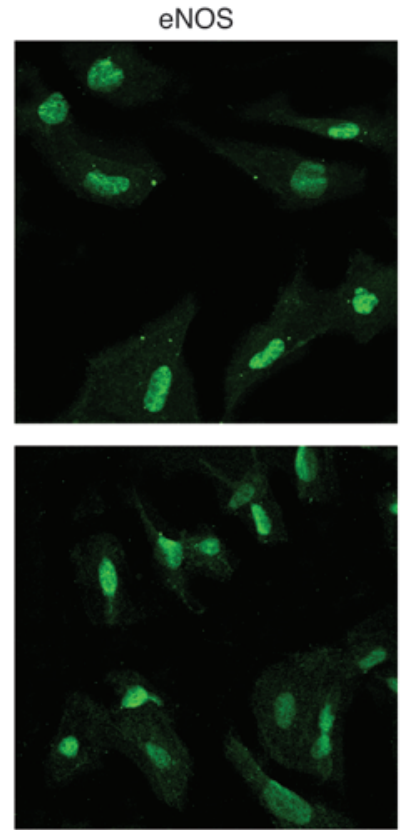

C38IM

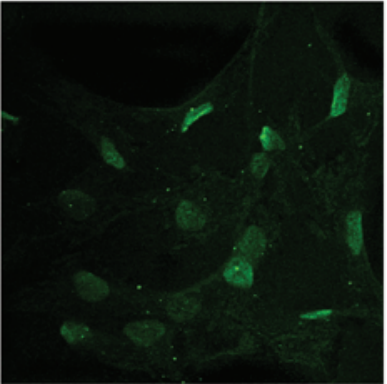

G2

С39IM
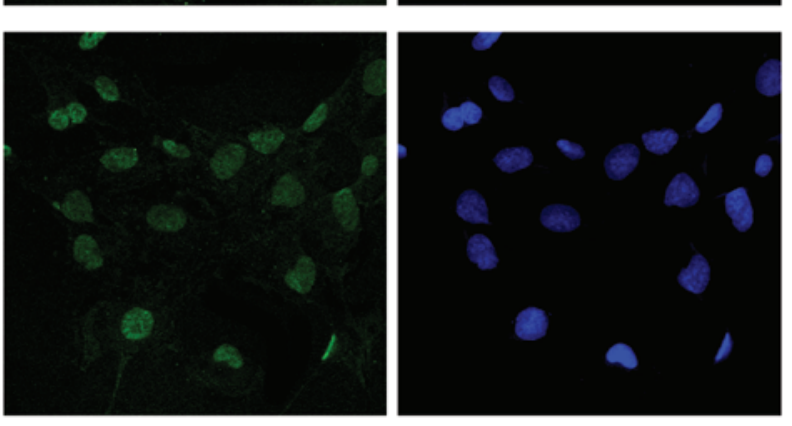

B

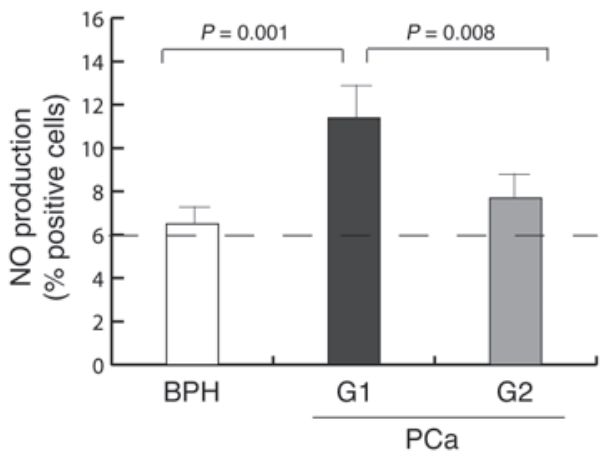

Topro 3
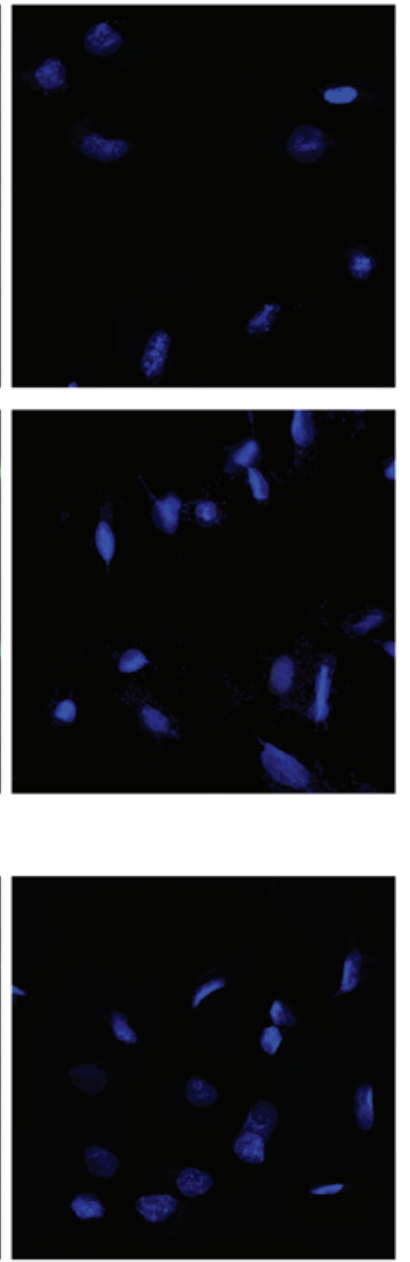

$\mathrm{PCa}$
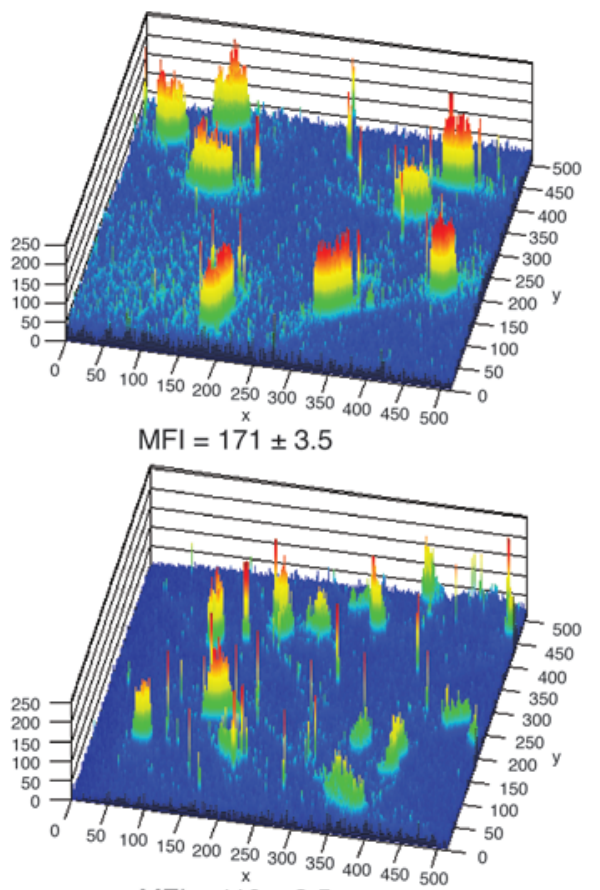

$\mathrm{MFI}=110 \pm 2.5$
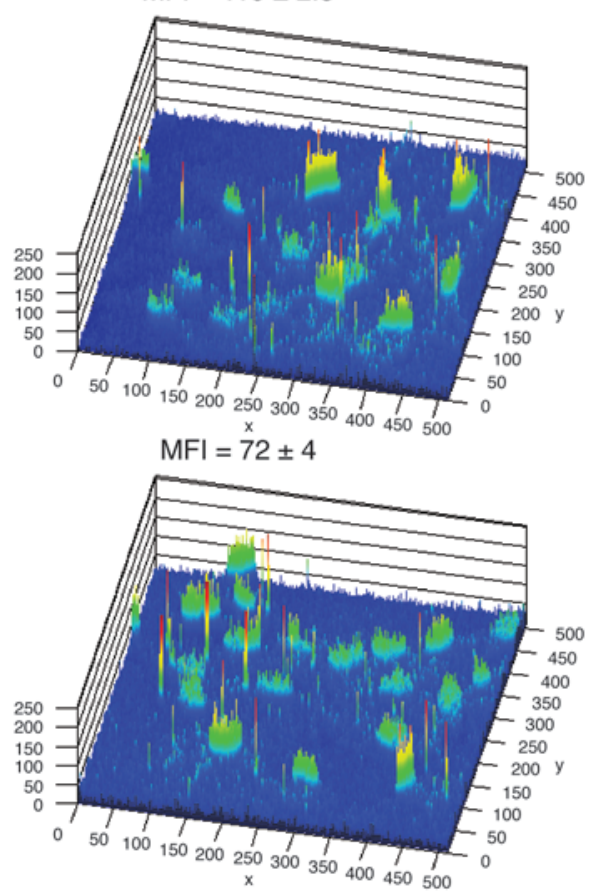

$\mathrm{MFI}=53 \pm 3$ 


\section{Figure 2}

Expression and activity of eNOS in PCa-derived cells. (A) G1 (C11IM and C27IM) and G2 (C38IM and C39IM) cells were stained with antibody to eNOS and examined by confocal microscopy. Nuclei were stained with Topro 3 . Original magnification, $\times 40$. The confocal images were digitally transformed using a computer program in order to map the MFI (in arbitrary units) for single eNOS-positive cells. The resulting $3 \mathrm{D}$ immunohistograms (right panels) revealed that eNOS protein was accumulated in the nuclei of prostate cells. The scale $0-250$ represents the MFI, with the colors blue, green, yellow, and red representing values in the 50-100, 100-150, 150-200, 200-250 range, respectively. (B) NO production was determined in cells from benign prostate hyperplasia $(\mathrm{BPH})(n=2)$ or PCa $(\mathrm{G} 1, n=9 ; \mathrm{G} 2, n=6)$, by DAF incorporation and FACS analysis (Supplemental Methods). Results of 3 independent experiments performed in duplicate (mean \pm SEM) are shown. Cell lines with a percentage of DAF-positive cells equal or less than $6 \%$ were considered negative (dashed line). Statistical significance is indicated.

in G2 cells (C38IM) in normoxia as detected by Western blot (Figure $1 \mathrm{E}$ ) and the present results (up to $10 \%$ of nuclei were positive in basal conditions) to the higher sensitivity of detection by confocal microscopy. The hypoxic phenotype of C27IM cells is shared by most of the cell lines belonging to the poor prognosis group (G1) and involves both HIF- $1 \alpha$ and HIF- $2 \alpha$ (data not shown). This phenotype, however, did not prevent a further significant increase in HIF- $1 \alpha /$ HIF- $2 \alpha$ expression, reaching almost $100 \%$ of nuclei, when cells were exposed to hypoxia (Figures 3 and 4). Indeed, confocal microscopy demonstrated a comparable response to hypoxia of both proteins in G1 and G2 cells (Figures 3 and 4).

Validation in vivo of the transcriptional prognostic signature. In order to validate in vivo the prognostic power of the gene expression profile of cultured PCa cells, TMAs were constructed from an independent retrospective cohort of patients with $\mathrm{PCa}(n=88)$, using paraffin-embedded tissue samples from the archive of the Pathology Department of the Regina Elena Cancer Institute. Samples were selected based on the longest follow-up period (12-14 years) and well-defined clinical outcome. The clinical and histopathological patient information is detailed in Supplemental Table 1. The poor prognosis group exclusively included patients deceased because of $\mathrm{PCa}$ or in clinical progression, defined by the presence of biochemical, local, or metastatic recurrence. TMA sections were stained with antibodies to HIF-1 $\alpha$, HIF- $2 \alpha$, or eNOS $(17,27)$ (Figure $5 \mathrm{~A})$. ER $\beta$ was included in the analysis because of its known role in PCa. The best results in terms of intensity and consistency were obtained with HIF- $2 \alpha$, eNOS, and ER $\beta$ antibodies. The HIF-1 $\alpha$ signal, instead, was mostly cytoplasmic and also only very weakly nuclear in clear cell renal carcinoma sections, which were used as positive controls. The quantitative statistical assessment of all scoring data, independently evaluated by 2 pathologists, demonstrated an association of HIFs, eNOS, and ER $\beta$ expression predominantly in tumors with poor prognosis. There was no association between expression of these markers and Gleason score.

The TMA analysis has revealed at least 3 major and interesting findings. (a) A highly significant correlation between expression of HIF-1 $\alpha$, eNOS (nuclear and cytoplasmic), and ER $\beta$ with diseasespecific survival (DSS) (Figure 5, B, D and E) was found. In particular, stronger staining for each of these molecules was independently associated with decreased survival (ER $\beta, P=0.0019 ; \mathrm{HIF}-1 \alpha$, $P=0.0484$; and eNOS, $P=0.0056$ ) as shown by Kaplan-Meier curves. (b) While no correlation was found between HIF-2 $\alpha$ expression and DSS, significant association between increased HIF- $2 \alpha$ nuclear staining and decrease of recurrence-free survival (local or metastatic,
$P=0.0015)$ was observed (Figure 5C). Further, high expression of HIF-2 $\alpha$ was significantly associated with increased biochemical recurrence $(P=0.0428$ and $P=0.0459$, respectively, for the American Society for Therapeutic Radiology and Oncology [ASTRO] consensus and the Phoenix definition; data not shown), suggesting a differential but critical role of HIF- $1 \alpha$ and HIF- $2 \alpha$ in clinical outcome. (c) Importantly, univariate analysis of ER $\beta$, HIF- $1 \alpha$, and eNOS (Figure $5 \mathrm{~F}$ ) revealed a direct correlation between increased expression of all 3 predictor variables and decreased DSS, with a much higher significance $(P=0.0001)$ than the individual variables. Moreover, combined expression of only ER $\beta$ and eNOS had sufficient predictive power on its own and even predicted patient survival with a higher significance than the Gleason score $(P<0.0001$ versus $P=0.0115$; Figure $5 \mathrm{G}$ and data not shown). The power of combined ER $\beta /$ eNOS expression as an independent prognostic factor emerged also in a multivariate analysis, comprising most of the classical markers of tumor aggressiveness, e.g., Gleason score, PSA, and pathological stage, albeit with reduced significance compared with the univariate analysis $(P=0.0066$ versus $P=0.0002$ for Gleason score and $P=0.0001$ for PSA). The pathological stage had no predictive power $(P=0.4195)$, most likely because of the relative homogeneity of the cohort (pathological stage T2 and T3).

Overall these findings confirm the strong prognostic relevance of the combined expression of eNOS, the hypoxia response, and ER $\beta$. Given the unexpected detection of eNOS in the nucleus of PCa cells in vitro (Figure 2), localization of the protein was examined also in the TMAs (Figure 6A). Nuclear eNOS was clearly detected in a large subset $(40 \%)$ of patients. Multivariate analysis revealed a very strong correlation between combined positivity for nuclear eNOS (independently of its expression levels) and HIF- $2 \alpha$ and all clinical end points (DSS, $P=0.0077$; biochemical recurrence, $P=0.0001$; metastatic recurrence, $P=0.0063$; and local recurrence free survival, $P=0.0006$ ) (Figure 6, B-E).

With the hazard ratios relative to the evaluated end points, Table 1 summarizes the contribution of each predictor variable examined in Figures 4 and 5.

In conclusion, the data from the scoring of TMAs derived from tissues from an independent cohort of patients are fully consistent with those obtained from the analysis of tumor cell populations, confirming the validity of the in vitro model that we have developed.

Chromatin remodelling upon estrogen and hypoxia stimuli at endogenous regulatory sequences of prognostic genes. Using ChIP, we previously observed a ligand-dependent involvement of both ERs in the transcriptional control of the bTERT promoter in the human PCa metastatic cell line $\mathrm{LNCaP}$ (2). Moreover, within the transcriptional profile of PCa-derived cells in vitro, we detected enhanced expression of genes involved in the hypoxia response (HIF1A, HIF2A, and ARNT) only in cells from patients with poor prognosis (2). Since $b T E R T$ is a direct target of both $\operatorname{ER}(2,31)$ and hypoxia signaling $(32)$, we hypothesized that HIFs might partner with ERs in PCa, through co-regulation of $h T E R T$. Given the role of eNOS in PCa, as evidenced by the TMA analysis, we also postulated a potential contribution of this protein to the ERs and HIFs functional partnership.

To investigate these hypotheses, we performed ChIP (see Supplemental Methods) under normoxia $\left(20 \% \mathrm{O}_{2}\right)$ or hypoxia $(1 \%$ $\left.\mathrm{O}_{2}\right)$, in the presence or absence of $17 \beta$-estradiol (E2) $\left(10^{-7} \mathrm{M}\right)$, using selected PCa cells from the G1 and G2 groups. Chromatins were immunoprecipitated by antibodies to ER $\beta, \mathrm{ER} \alpha, \mathrm{HIF}-1 \alpha$, HIF- $2 \alpha$, HIF- $1 \beta$, or eNOS, and DNA sequences proximal to or encompassing estrogen and hypoxia response elements within 
A
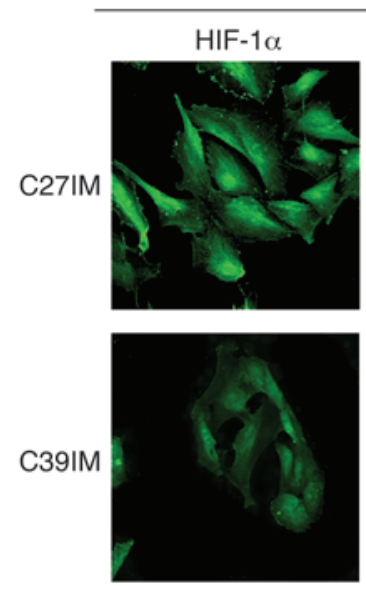

Normoxia
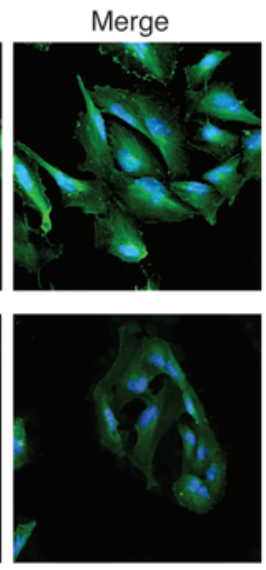

B
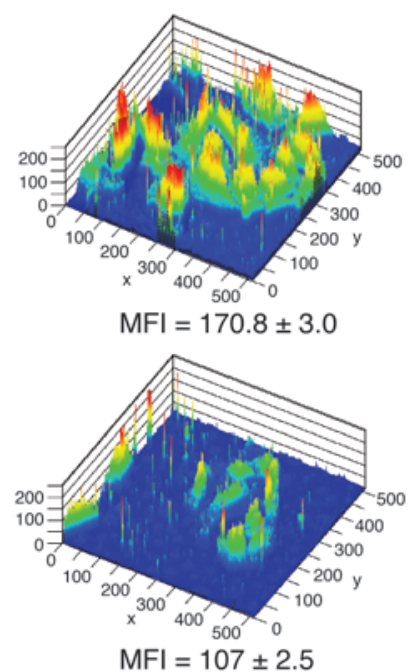

$\mathrm{MFI}=107 \pm 2.5$

Hypoxia
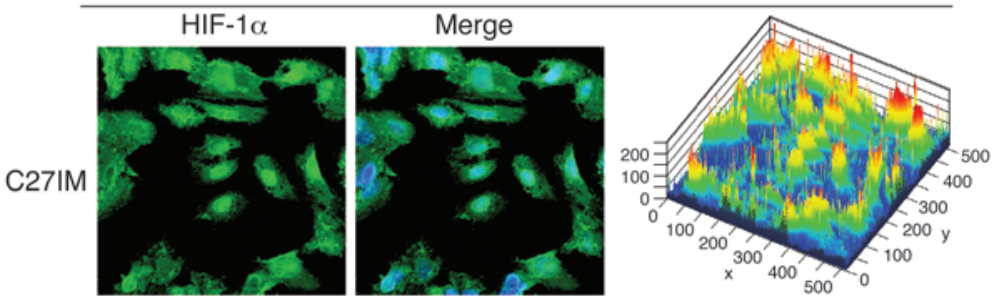

$\mathrm{MFI}=205.1 \pm 3.5$
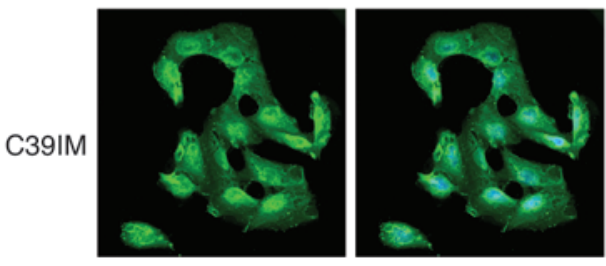

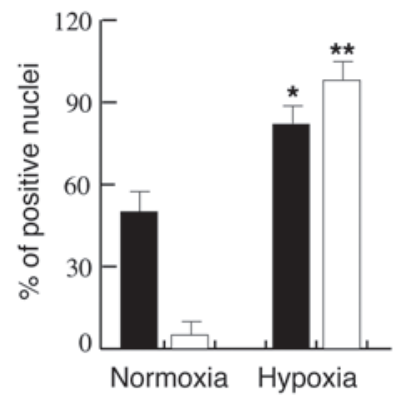

Normoxia Hypoxia
C27IM C39IM

\section{Figure 3}

Nuclear localization of HIF-1 $\alpha$. (A) C27IM and C39IM under normoxia $\left(20 \% \mathrm{O}_{2}\right.$; left panels) or hypoxia ( $1 \% \mathrm{O}_{2}$; right panels) were fixed and stained with anti-HIF- $1 \alpha$ antibody and examined by confocal microscopy. Nuclei were stained with Topro 3 . Original magnification, $\times 40$. Individual and merged fluorescence signals are shown. The fluorescing capability of cells in each condition was defined as MFI. Images are from a typical experiment out of 2 performed. (B) The percentage of the HIF-1 $\alpha$-positive nuclei was obtained from confocal images by counting nuclear positive cells among 200 per sample. Images shown are representative of all examined fields. Results shown are the mean of 2 independent experiments \pm SEM. ${ }^{*} P<0.05 ;{ }^{* *} P<0.001$.

a $10-\mathrm{kb}$ region of the endogenous $h T E R T$ regulatory sequences were amplified (Figure 7A).

Optimal hypoxic conditions were defined by treating cells over time with E2, hypoxia, or both and evaluating induction of the HIF- $1 \alpha$ protein at times known to be optimal for the ER cyclic occupancy in vivo of target promoters (refs. 33-35 and data not shown). Western blots on nuclear extracts from most G1 and G2 cell lines showed that the HIF- $1 \alpha$ signal generally peaked at 135 minutes of exposure to $1 \% \mathrm{O}_{2}$, a concentration within the range observed in solid tumors (refs. 36 and 37 and data not shown). Therefore, this condition was used in further ChIP.

Substantial differences emerged in the behavior of the proteins in G1 (C27IM) and G2 (C38IM) cells (Figure 7B). Regardless of the stimulus (E2 or hypoxia, alone or combined), eNOS was recruited to all analyzed sites of the $b T E R T$ promoter only in G1 cells. Recruitment upon E2 treatment (about a 6-fold increase over control) was higher than under hypoxia (3- to 4-fold increase over control), and maximum recruitment occurred upon the combined stimuli (7- to 10 -fold increase with a peak on site III). In comparison, recruitment in G2 cells was, at most, 2-fold increase over control.

Occupancy of the $b T E R T$ promoter sites by ER $\beta$ or HIFs, on the other hand, was more strongly dependent upon the stimulus and the cell context. Upon treatment with only estrogen, we observed ER $\beta$ but not ER $\alpha$ occupancy (about a 4-fold increase over control) of the bTERT sites III and IV in C27IM (G1) cells and II and III in C38IM (G2) cells. Unexpectedly, estrogen treatment also induced 
A
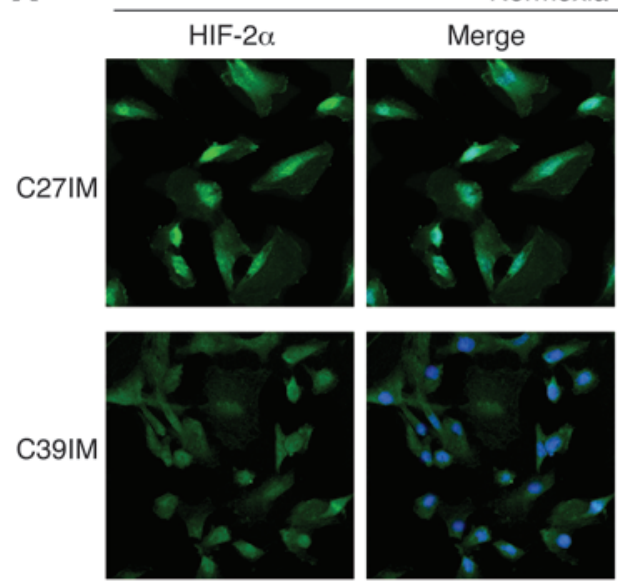

Normoxia

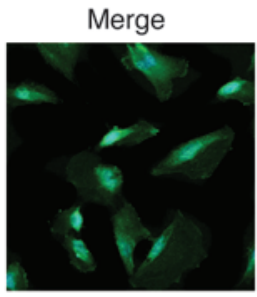

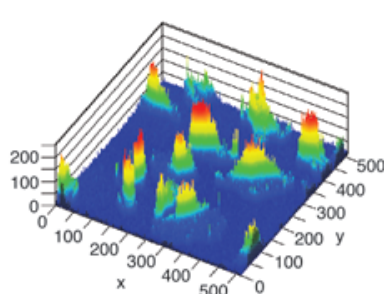

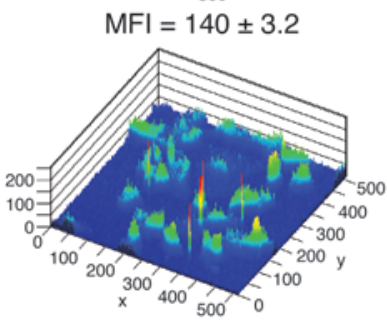

$\mathrm{MFI}=75 \pm 3.1$

Hypoxia
B

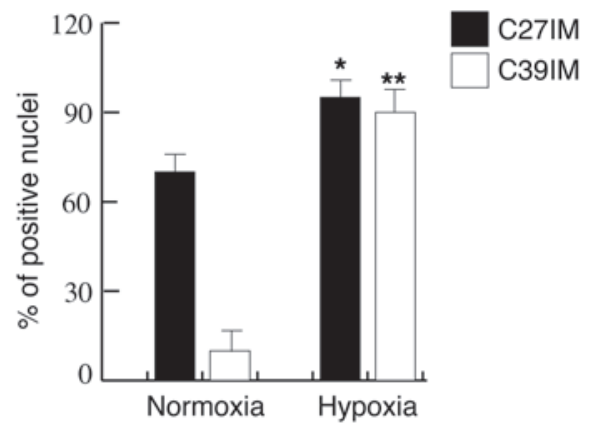

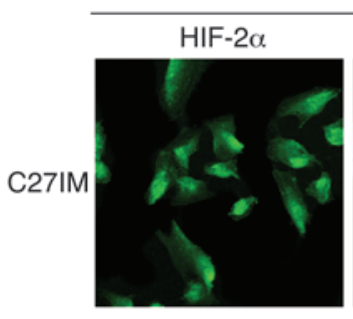

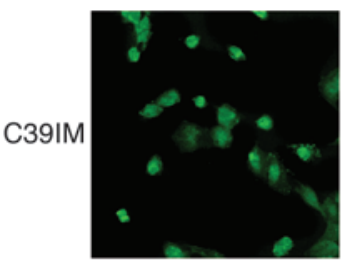

\section{Merge}
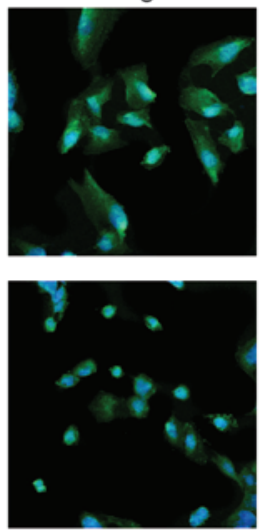

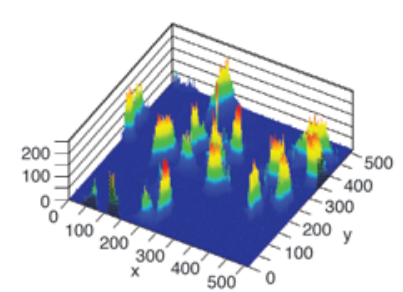

$\mathrm{MFI}=175 \pm 4$

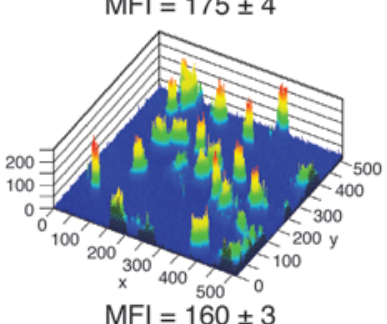

Figure 4

Nuclear localization of HIF-2 $\alpha$. (A) C27IM and C39IM under normoxia $\left(20 \% \mathrm{O}_{2}\right.$; left panels) or hypoxia ( $1 \% \mathrm{O}_{2}$; right panels) were fixed and stained with anti-HIF-2 $\alpha$ antibody and examined by confocal microscopy. Nuclei were stained with Topro 3 . Original magnification, $\times 40$. Individual and merged fluorescence signals are shown. The fluorescing capability of cells in each condition was defined as MFI. Images are from a typical experiment out of 2 performed. (B) The percentage of the HIF-2 $\alpha$-positive nuclei was obtained from confocal images by counting nuclear positive cells among 200 per sample. Images shown are representative of all examined fields. Results shown are the mean of 2 independent experiments \pm SEM. ${ }^{*} P<0.05 ;{ }^{* *} P<0.001$.

recruitment of HIFs, primarily HIF- $1 \alpha$ in C27IM cells and HIF- $2 \alpha$ in C38IM cells (3- and 4-fold increase, respectively) with the same pattern as ER $\beta$. Hypoxia caused enrichment of HIF-1 $\alpha$ in C27IM cells and HIF- $2 \alpha$ in C38IM cells, with maximum enrichment (up to a 6-fold increase) at site III in both cell lines. Notably, combined E2 and hypoxia markedly increased (to about a 12-fold increase) recruitment of both HIF- $1 \alpha$ and ER $\beta$ at site III exclusively in C27IM cells. Moreover, while HIF-2 $\alpha$ recruitment in C27IM cells was constant at site III (about a 3-fold increase) upon single or combined treatments, in C38IM cells, maximum recruitment (7-fold increase) occurred under hypoxia, and combined treatment reduced it to levels observed with E2 alone (4-fold increase). Occupancy by HIF-1 $\alpha$ in C38IM cells or by ER $\beta$ or HIF- $1 \alpha$ in both cell lines was absent or minimal under all conditions (Figure 7B and data not shown).
In keeping with these results, substantial recruitment of HIF- $1 \alpha$, ER $\beta$, and eNOS was also found, exclusively in G1 cells, on promoters of other prognostic genes, e.g., the MSH2 gene involved in mismatch repair and the cyclin B1 (CCNB1) and the PS2 genes (Supplemental Figure 1).

To assess the specificity of our findings, ChIPs were performed in von Hippel-Lindau-deficient renal carcinoma cells (786-0) lacking HIF-1 $\alpha$ expression but overexpressing HIF- $2 \alpha$. As expected, no recruitment of HIF- $1 \alpha$ or endogenous ER $\beta$ was observed at site III of the $b T E R T$ promoter upon combined treatment (data not shown).

Formation of combinatorial complexes between ER $\beta$ and eNOS or HIFs. We next asked whether ER $\beta$, HIF- $1 \alpha$, or HIF- $2 \alpha$ and eNOS form combinatorial complexes on chromatin. Serial ChIPs were performed in PCa cells exposed to E2 or hypoxia, alone or combined. 
A
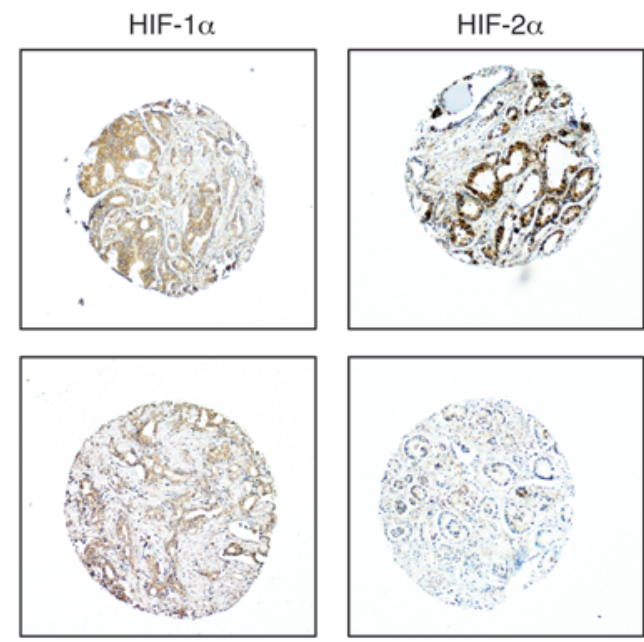

B

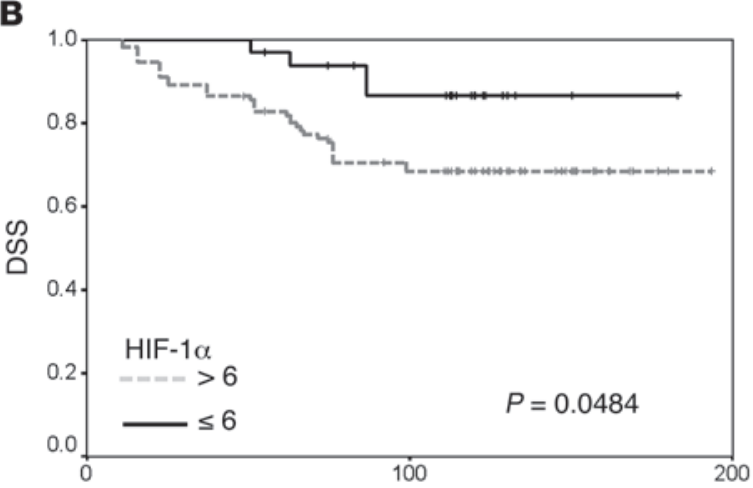

D

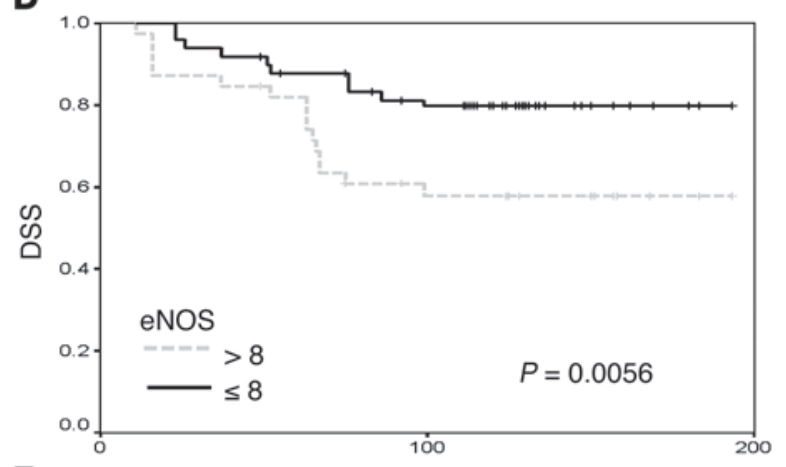

F

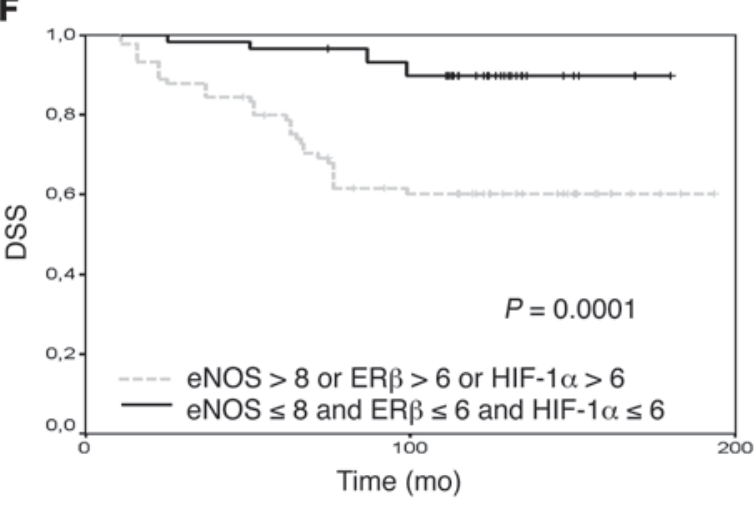

eNOS
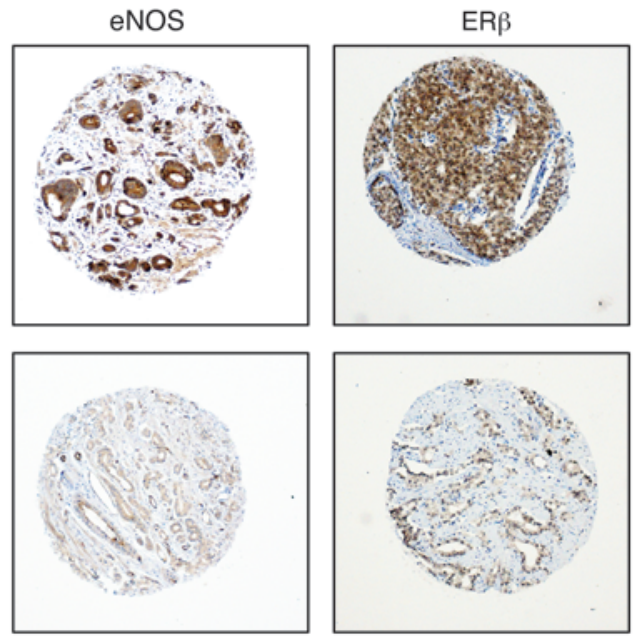

C

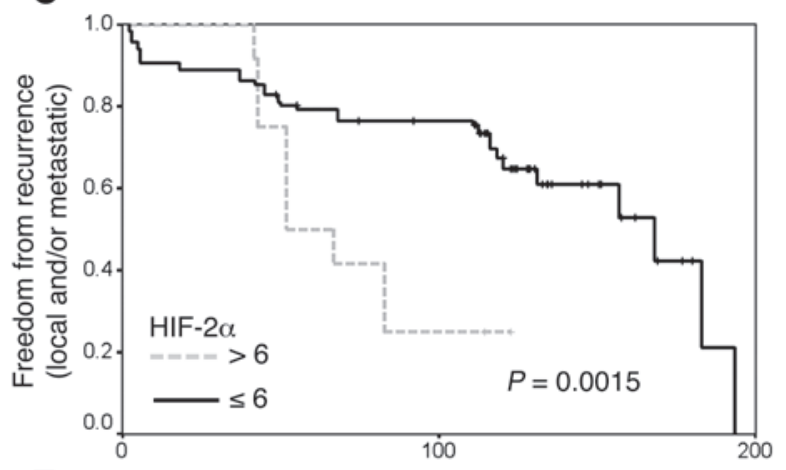

E

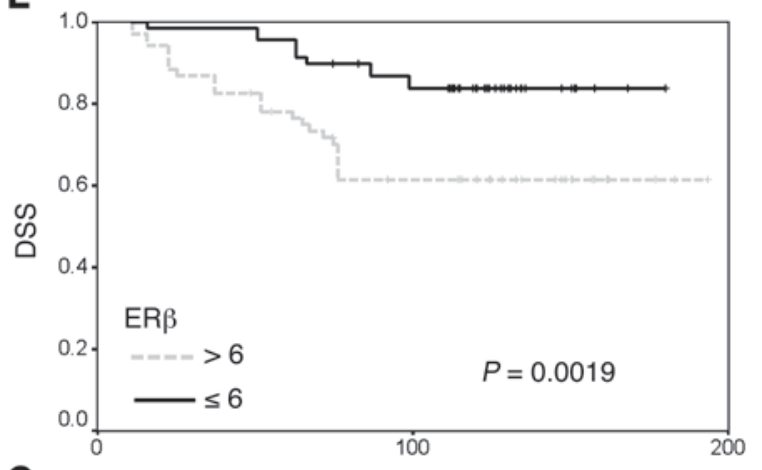

G

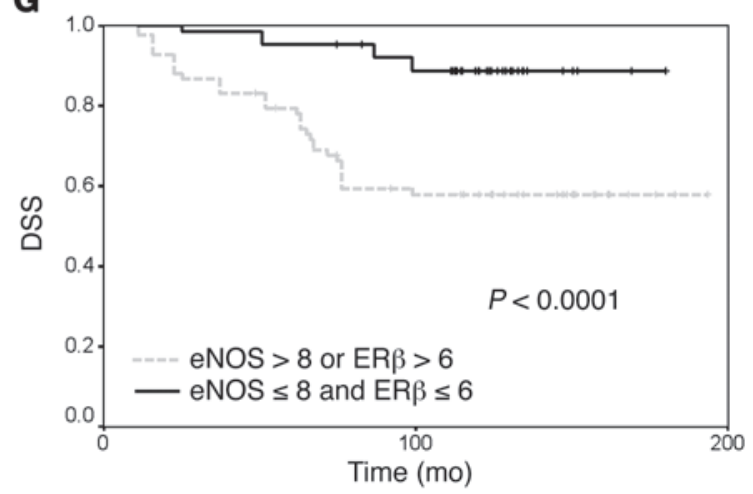




\section{Figure 5}

PCa TMA. (A) Representative TMA cores immunostained for HIF-1 $\alpha$, HIF-2 $\alpha$, eNOS, or ER $\beta$. Tumor sections with comparable Gleason score were selected, and examples of tumor with high (top row) or low (bottom row) protein expression, corresponding to poor or good prognosis, are shown. Original magnification, $\times 10$. (B-E) Kaplan-Meier DSS curves for patients with PCa with low $(I \times Q \leq 6$ and $I \times Q \leq 8$; see Methods) or high $(I \times Q>6$ and $I \times Q>8)$ expression levels of $\mathrm{HIF}-1 \alpha$, eNOS, or ER $\beta$ (B, D, and E, respectively), and Kaplan-Meier curve of recurrence-free survival for patients with PCa with low $(I \times Q \leq 6)$ or high $(I \times Q>6)$ expression levels of HIF-2 $\alpha(\mathbf{C})$. (F and $\mathbf{G})$ KaplanMeier DSS curves for patients with $\mathrm{PCa}$ with expression level under the threshold [eNOS $\leq 8, \mathrm{ER} \beta \leq 6$, and HIF- $1 \alpha \leq 6(\mathrm{~F})$; eNOS $\leq 8$ and $E R \beta \leq 6(\mathbf{G})$ ] or over the threshold for at least 1 protein $[(\mathrm{eNOS}>8$, $\mathrm{ER} \beta>6$, or HIF- $1 \alpha>6(\mathbf{F})$; eNOS $>8$ or $\mathrm{ER} \beta>6(\mathbf{G})]$.

Cross-linked chromatin was immunoprecipitated with antibodies to HIF- $1 \alpha$, HIF- $2 \alpha$, or eNOS, then reimmunoprecipitated with antibody against ER $\beta$ (or vice versa). eNOS/ER $\beta$ and HIFs/ER $\beta$ complexes were all strongly enriched along the $h T E R T$ regulatory region, but with distinct dynamics, exclusively in C27IM cells (Figure 8). Specifically, recruitment of the eNOS/ER $\beta$ complex, which occurred only at site III and in similar amounts upon normoxia or hypoxia, was enhanced at this site and present also at site I upon E2 treatment or treatment with E2 plus hypoxia. On the other hand, the HIF-1 $\alpha /$ ER complex was recruited only at site III upon hypoxia but at all sites upon combined treatment, while the HIF- $2 \alpha / E R \beta$ complex was present at all sites in normoxia but was removed from site III by every treatment. Therefore, occupancy of this ER binding site by HIF- $1 \alpha / E R \beta$ or HIF- $2 \alpha / E R \beta$ complexes appears mutually exclusive, suggesting that $\operatorname{ER} \beta$ can interact on chromatin with both HIFs, but that, upon combined treatment, the HIF-1 $\alpha / \mathrm{ER} \beta$ complex is highly favored for site III occupancy. The absence of complexes at any site in C38IM cells underlines again the difference between G1 and G2 cell lines.

Finally, coimmunoprecipitation of soluble proteins revealed that the eNOS/HIF- $1 \alpha$ and HIF- $1 \alpha /$ ER $\beta$ complexes also form in the absence of DNA (data not shown). We were, however, unable to determine whether this was the case for the eNOS/ER $\beta$ interaction. In addition to technical reasons related to performance of available antibodies, this negative result may be due to the fact that interaction between these proteins is inherently weak and is stabilized by contacts with the cognate DNA binding sites (estrogen-responsive elements [EREs]).

Function of combinatorial complexes on estrogen and/or bypoxia-responsive elements. To address whether recruitment of eNOS, as assessed by ChIP assays, is capable of transactivating estrogen- and/or hypoxia-responsive gene promoters (and requires intact ERE and hypoxia-responsive element [HRE] binding sites), we assessed the effects of eNOS on luciferase activity driven by the $b T E R T$ promoter with intact (P-1009) or mutated ERE (P-1009Mut), in the presence of ligand-activated ER $\beta$ or driven by the hypoxia-dependent erythropoietin (EPO) gene promoter (with intact or mutated HRE) upon HIF- $1 \alpha /$ HIF- $2 \alpha$ activation. The results (Supplemental Figure 3 , A and B) clearly indicate that overexpression of eNOS, caused by cotransfection of a constitutively active eNOS (S1177D), induced P-1009 luciferase by approximately an 2-fold increase over basal activity and strongly potentiated it ( $\sim 5$-fold increase) upon overexpression of ER $\beta$. Conversely, both effects were virtually absent on the STERT promoter with mutated ERE (P-1009Mut). In addition, complete abrogation of luciferase activity (basal and induced) was brought about by pharmacological inhibition of PI3K/AKT using an AKT inhibitor, demonstrating the requirement for AKT-mediated phosphorylation of eNOS in the hTERT promoter activity. Overexpression of eNOS also significantly $(P<0.05)$ induced the EPO reporter, a 5-fold increase, over basal activity and strongly enhanced it by a 10- or 24-fold increase upon cotransfection of HIF-1 $\alpha$ or HIF- $2 \alpha$, respectively. Again the effect was virtually abolished by using the HRE-mutated EPO reporter (EPOMut), confirming that synergism between eNOS and HIFs requires an intact HRE.

Modulation of target gene expression. We next evaluated mRNA expression of classical gene targets of HIFs or ERs (e.g., VEGF, human VEGF type 2 receptor [ $h K D R]$, and GLUT1). As shown in Figure 9A, under basal conditions, all mRNA levels were significantly higher in G1 (C27IM) than in G2 (C38IM) cells $(P=0.029$ for $h K D R ; P=0.014$ for VEGF; and $P=0.013$ for GLUT1) in agreement with results from primary (2) and immortalized cells (Figure 1D). Expression was enhanced by hypoxia to a comparable extent in both cell lines, demonstrating that the response to low oxygen tension is intact even in G2 cells. Most interestingly, and in keeping with the ChIP data, we observed strong induction of these mRNAs upon treatment with E2 plus hypoxia only in G1 cells.

A similar transcriptional pattern was detected also in a different cell type, human umbilical vein endothelial cells, a well-characterized model for the hypoxia-response expressing endogenous ER (38), which is responsive to estrogen (data not shown). Separate $\mathrm{E} 2$ or hypoxia stimuli substantially increased mRNAs of $h K D R$, GLUT1, MMP9, and $b T E R T$ by 4 hours after treatment (Supplemental Figure 2, A and B). Notably, combined stimuli accelerated this effect to within 2 hours after treatment, and synergistically enhanced recruitment of HIF- $1 \alpha$ to the $h T E R T$ and VEGF promoters (Supplemental Figure 2C). No HIF-1 $\alpha$ occupancy of the control cJun promoter was detected (data not shown).

Reciprocal conversion of the transcriptional profile of G1 and G2 cells. The striking differences in the estrogen- and hypoxia-dependent chromatin remodeling by combinatorial complexes among eNOS, HIFs, and ER $\beta$ observed in G1 and G2 cells, prompted us to investigate whether modulation of these factors by genetic or pharmacological approaches could alter the cell molecular phenotype, i.e., the transcription profile of prognostic genes.

G2 cells, derived from patients with good prognoses, were transfected with the constitutively active forms of HIF-1 $\alpha$ or eNOS or treated with the NO donor DETA-NO, and transcription of the target genes $h K D R, G L U T 1$, and VEGF was measured by quantitative RT-PCR (Figure 9, B and C). Basal levels of gene expression (Figure 9B) were significantly increased by all 3 approaches. In addition, upon transfection of active eNOS (Figure 9C), cells acquired responsiveness to $\mathrm{E} 2$, hypoxia, or their combination. In all respects, therefore, $\mathrm{G} 2$ cells mimicked the transcription profile of G1 cells.

Reciprocal conversion of the G1 to the $\mathrm{G} 2$ transcriptional profile was achieved by treating G1 cells with the NOS inhibitors 7-nitroindazole $(7 \mathrm{~N})$ or $\mathrm{N}(\mathrm{G})$-nitro-L-arginine methyl ester (L-NAME), both of which resulted in significant transcriptional downregulation of the target genes (Figure 9D). Most interestingly, in G1 cells exhibiting constitutive phosphorylation of eNOS and AKT, pharmacological interference with NO production or overexpression of a dominant-negative mutant of eNOS (eNOS S1177A) caused a marked reversal of the constitutive hypoxic phenotype, as shown by decreased HIF-1 $\alpha$ and HIF-2 $\alpha$ expression (Figure 9E), demonstrating the existence of a regulatory loop between $\mathrm{NO}$ production and the hypoxia signaling. 
A
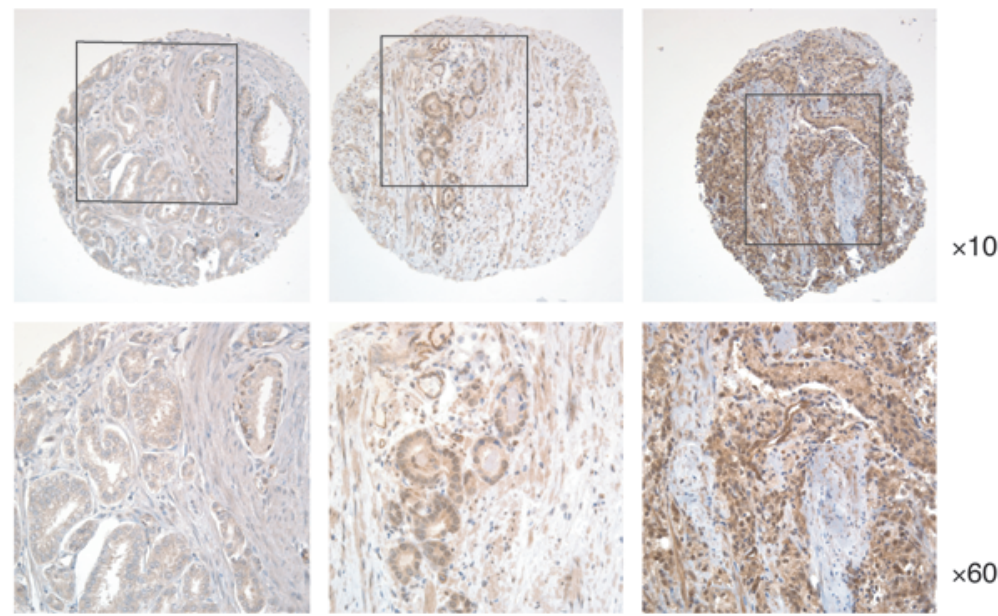

$\times 60$

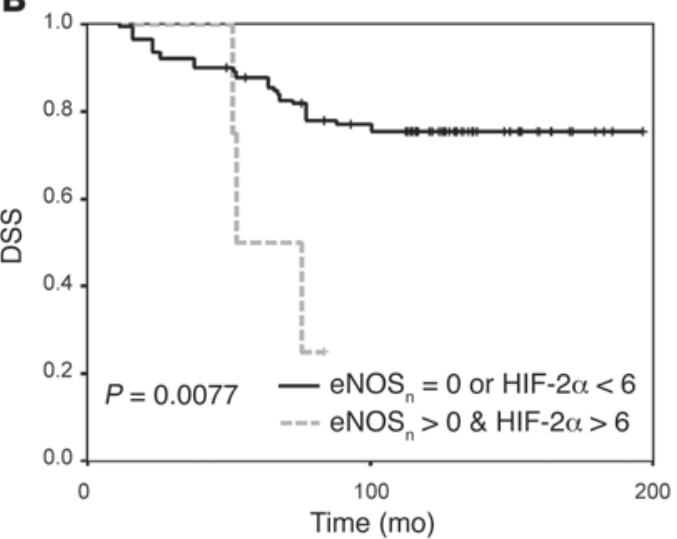

D

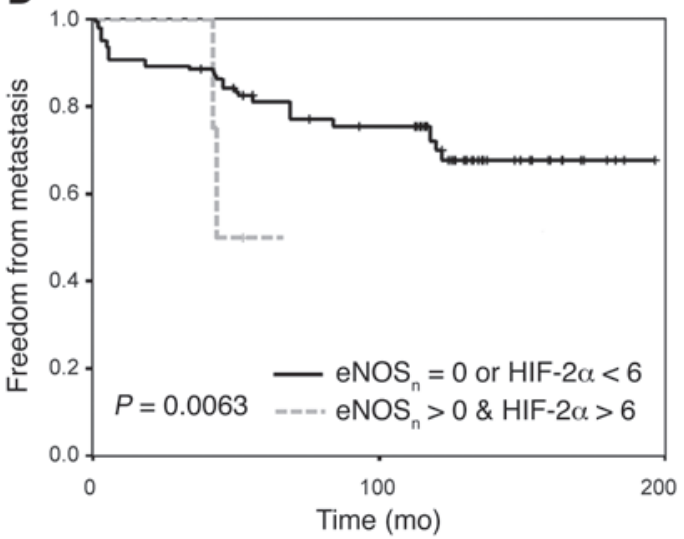

C

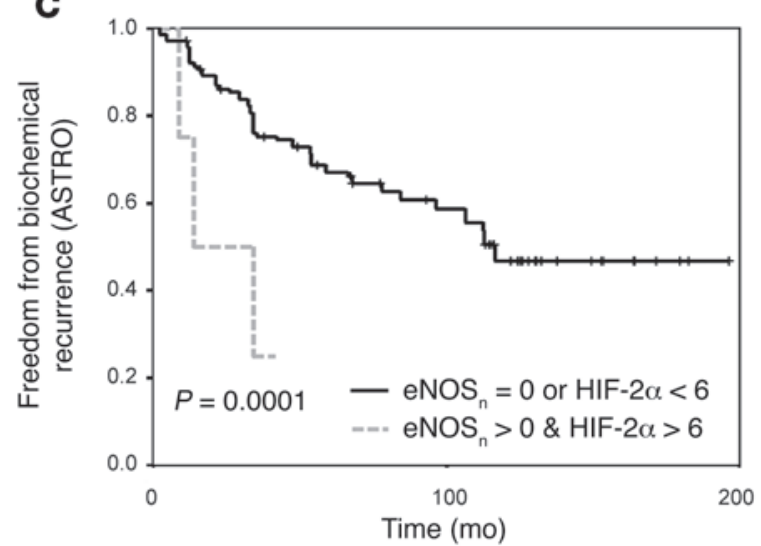

E

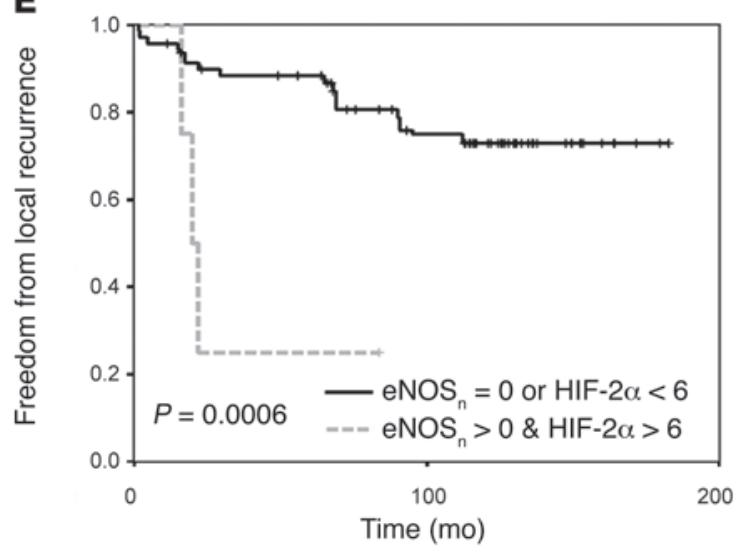

Figure 6

Nuclear localization of eNOS in TMA. (A) Nuclear localization of eNOS (eNOS $n$ ) was examined in TMA cores immunostained with antibody to the protein as in Figure 5. Representative cores of tumors with different degrees of eNOS expression and their magnification are shown. The left-most core is an example of negative tumor and positive basal cells in the normal tissue. Basal cell positivity served as internal control for the scoring. (B-E) Kaplan-Meier curves for DSS (B) and freedom from biochemical (C), metastatic (D), or local recurrence (E) for patients with PCa with positivity for nuclear eNOS and expression levels of HIF-2 $\alpha$ below or above threshold. Biochemical recurrence was defined according to the American Society for Therapeutic Radiology and Oncology (ASTRO) consensus definition as described in Methods.

\section{Discussion}

The results presented here support the hypothesis that the more aggressive prostate tumors are characterized by concomitant abnormal activation of multiple signaling pathways, particu- larly those mediated by the ER, the HIFs, and NOS. Specifically, our results reveal the formation and prognostic power for PCa of combinatorial complexes of ER $\beta$ with eNOS and/or HIF- $1 \alpha$ and HIF- $2 \alpha$, and their enhanced co-recruitment on chromatin upon 


\section{Table 1}

Significance and hazard ratio of Kaplan-Meier analysis of TMA data

\begin{tabular}{|c|c|c|c|}
\hline End point & Biomarker & $P A$ & Hazard ratio (95\% confidence interval) \\
\hline \multirow[t]{4}{*}{ DSS } & $\mathrm{ER} \beta$ & 0.0019 & $0.3469(0.1795-0.6793)$ \\
\hline & HIF-1 $\alpha$ & 0.0484 & $2.726(1.0054-4.7665)$ \\
\hline & $\mathrm{eNOS}_{\mathrm{c}}>8$ & 0.0056 & $0.4065(0.1572-0.7287)$ \\
\hline & eNOS $_{n}>0$ and HIF2 $\alpha>6$ & 0.0077 & $0.2328(0.0042-0.4356)$ \\
\hline \multirow[t]{2}{*}{ Metastasis } & HIF- $2 \alpha$ & 0.0362 & $0.4100(0.0751-0.9175)$ \\
\hline & eNOS $_{n}>0$ and HIF- $2 \alpha>6$ & 0.0063 & $0.2278(0.0036-0.3959)$ \\
\hline Metastasis and/or local recurrence & HIF- $2 \alpha$ & 0.0015 & $0.3397(0.0535-0.4992)$ \\
\hline Local recurrence & eNOS $_{n}>0$ and HIF- $2 \alpha>6$ & 0.0006 & $0.1666(0.0006-0.1315)$ \\
\hline \multirow[t]{2}{*}{ Biochemical recurrence ${ }^{B}$} & HIF- $2 \alpha$ & 0.0428 & $0.5083(0.1650-0.9710)$ \\
\hline & eNOS $_{n}>0$ and HIF- $2 \alpha>6$ & 0.0001 & $0.1779(0.0011-0.1102)$ \\
\hline
\end{tabular}

Alog-rank test; BAccording to the American Society for Therapeutic Radiology and Oncology (ASTRO) consensus definition. c, cytoplasmic; n, nuclear.

combined estrogen and hypoxia stimuli. These results are validated by the data obtained in vivo on TMAs, demonstrating the very high predictive power of (a) the combined enhanced expression of ER $\beta$ and eNOS in terms of DSS and (b) the association between nuclear localization of eNOS and enhanced expression of HIF- $2 \alpha$ for all clinical end points. These findings represent a substantial advance toward anticipation of diagnosis and better refinement of prognosis. Indeed, routine assessment of these biomarkers, particularly the combination of ER $\beta$ and eNOS and of nuclear eNOS and HIF-2 $\alpha$, by traditional immunohistochemistry, as early as at the time of biopsy, should contribute to increase the accuracy of PCa patient stratification.

The finding, which we believe to be novel, of a functional crosstalk among ER $\beta$, HIFs, and eNOS provides an intriguing alternative interpretation of PCa pathogenesis and progression. Tumors with a constitutively hypoxic phenotype and with enhanced production of NO and eNOS nuclear localization (as is the case of G1 cells), may be more sensitive to changes in the prostate microenvironment, such as those determined by estrogen signaling or estrogen/androgen metabolites. Thus, upon exposure to estrogen they may acquire a more malignant phenotype, enabling colonization at distant sites. Our previous study (34) has indeed outlined the relevance of intracellular conversion of androgens to estrogens in the etiopathogenesis of PCa, by demonstrating that aromatase inhibitors reduce telomerase activity, an early marker of prostate carcinogenesis. Of relevance to this line of arguing is that ER $\beta$ is the first ER expressed in the fetal prostate and the only ER present in epithelial and stromal cells during early ductal morphogenesis $(10,11)$. We can therefore assume that, with the AR, ER $\beta$ mediates the very early stage of fetal prostate development, suggesting its key role in hormonebased chemoprevention strategies for PCa. Our observation that ER $\beta$ forms functional complexes with eNOS on chromatin and that combined overexpression of the 2 proteins strongly potentiates $h T E R T$ promoter activity opens new perspectives about the role of estrogens and NO in PCa. The presence of eNOS in the tumor cell nuclei may in fact influence the specificity of estrogen action on selected genes that in turn may contribute to tumor maintenance and progression $(18,25)$. Interestingly, binding to DNA, indeed the integrity of the ERE binding site, appears to be a conditio sine qua non for the formation of the complex and therefore, for the execution of eNOS nuclear function (see Supplemental Figure 3). This requirement may explain our failure to coim- munoprecipitate the eNOS/ER $\beta$ complex in the absence of DNA (data not shown). Stabilization of complexes between the ER and other transcription factors by contacts with the cognate DNA response elements (EREs) has been previously reported (39).

In addition to its functional interaction with ER, eNOS acts as downstream effector of the hypoxia-activated response (17). Numerous reports have assigned to HIFs an essential role in the progression of solid tumors $(4,30,40)$, including a role in the lethal phenotype of endocrine cancers (41). Our in vitro and in vivo results confirm and reinforce this role. Within the transcriptional signature with prognostic value we previously described (2), HIFs emerged as a predominant functional class of genes upregulated in cells from patients with poor prognosis (G1). These cells exhibit a constitutively hypoxic phenotype but retain responsiveness to both estrogen and hypoxia. Indeed, upon the combined stimuli, we observed chromatin remodeling by HIFs/ ER $\beta$ and eNOS/ER $\beta$ complexes, accompanied by enhanced transcription of prognostic estrogen and hypoxia target genes. Interestingly, this transcription pattern mimics that observed in human endothelial cells (Supplemental Figure 2) in response to estrogen and hypoxia, in agreement with the more undifferentiated phenotype of G1 as compared with G2 cells.

In vivo, high expression of HIF- $1 \alpha$ or HIF- $2 \alpha$ on their own is predictive of poor outcome. However, this predictive power is significantly potentiated by the concomitant expression of ER $\beta$ and eNOS in the case of HIF- $1 \alpha$ and especially of nuclear eNOS in the case of HIF- $2 \alpha$ (Figures 5 and 6 and Table 1). In this regard, it should be emphasized that, rather than the recruitment of a single protein, the crucial event in determining the tumor phenotype and ultimately affecting clinical outcome appears to be the dynamic assembly on chromatin of at least 2 proteins with prognostic value. Specifically, formation of the ER $\beta /$ eNOS, ER $\beta$ / HIF- $1 \alpha$, and ER $\beta /$ HIF- $2 \alpha$ combinatorial complexes, as demonstrated by serial ChIP (Figure 8), is virtually negligible in C38IM cells, under any treatment evaluated, as compared with C27IM cells. These findings reiterate the importance of eNOS, specifically the eNOS-associated complexes and particularly eNOS/ER $\beta$ and eNOS/HIF- $2 \alpha$, in the progression of PCa.

The relevant role of eNOS is further supported by our findings that modulation of eNOS expression and activity is capable of reverting the transcriptional pattern of tumor cells with opposite phenotype (G1 and G2 cells; Figure 9, B-E). Specifically, overexpression of eNOS or treatment with a NO donor confers to con- 
A hTERT
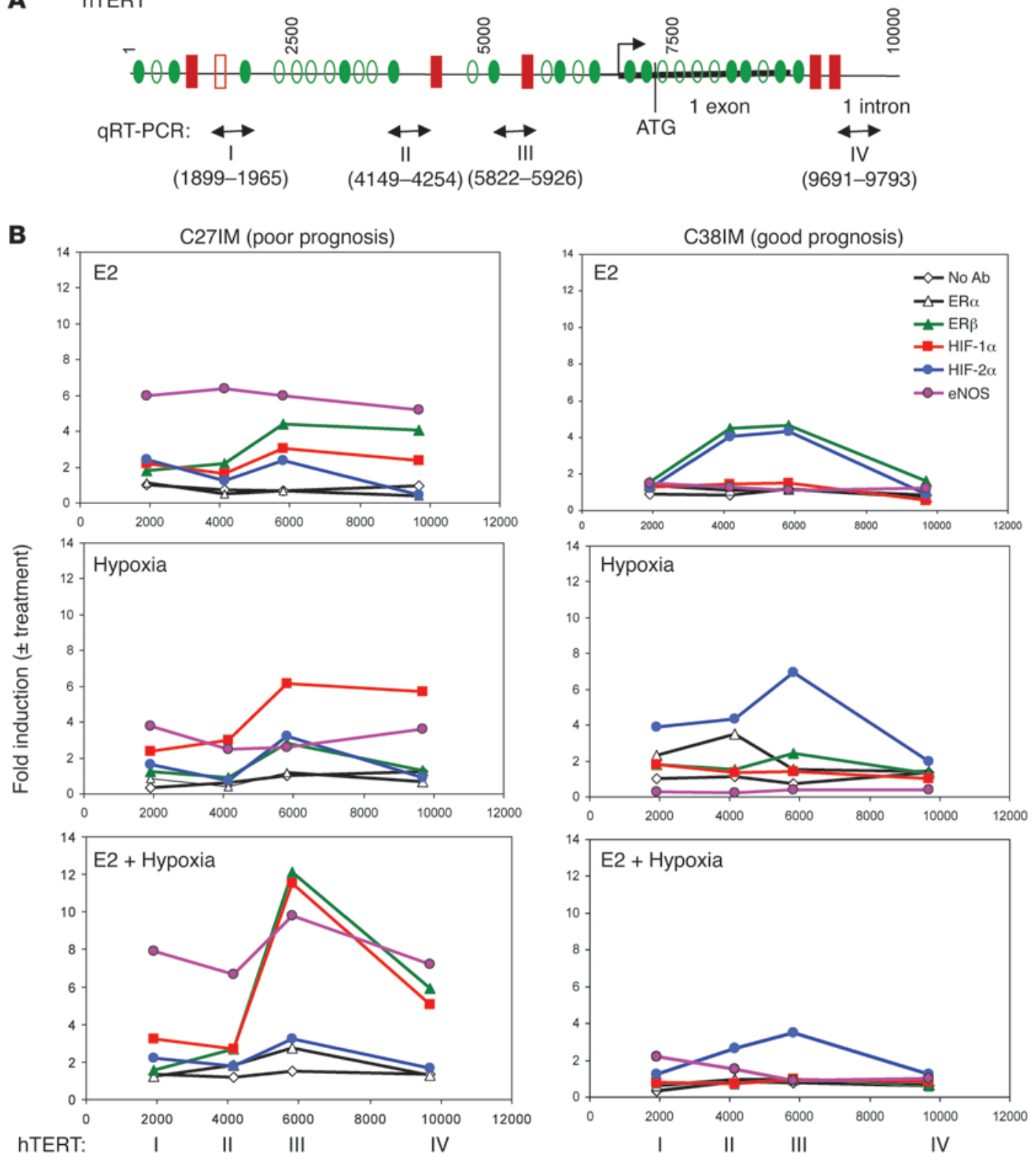

Figure 7

ChIPs onto the hTERT promoter. (A) Location of putative transcription factor binding sites and predicted transcription start site (arrow) in the promoter region of hTERT $(10 \mathrm{~kb})$ identified with the MatInspector database. ATG, exon 1, and partial intron 1 are indicated; double-arrowed lines identify the regions I, II, III, and IV amplified in ChIP by quantitative RT-PCR; numbers in parenthesis indicate $5^{\prime}$ primer ends. Arabic numbers refer to the length of the fragment in base pairs. Closed rectangles, EREs; open rectangles, estrogen-related receptor binding sites; closed ovals, HIF-1 $\alpha$ binding sites; open ovals, ARNT (HIF-1 $\beta$ ) heterodimers/dioxin receptor binding sites. (B) ChIP experiments were performed in parallel using immortalized PCa cancer cells from the G1 and G2 groups (C27IM and C38IM, respectively). Cells were treated with or without E2 (10-7 M), in normoxia or hypoxia $\left(1 \% \mathrm{O}_{2}\right)$, alone or in combination for 135 minutes. Immunoprecipitations were performed using antibodies to ER $\alpha$, ER $\beta$, HIF-1 $\alpha$, HIF-2 $\alpha$, eNOS, or no antibody (NoAb) as negative control. Data are represented as fold induction over control (vehicle alone in normoxia); Arabic and roman numbers are as in A. Recruitment of each transcription factor onto the hTERT promoter was detected by quantitative RT-PCR using primers specific for region I, II, III, and IV. The data represent the mean of 3 independent experiments.

stitutively unresponsive G2 cells the responsiveness to estrogen and hypoxia typical of G1 cells (Figure 9, B and C). Conversely, interference with NO synthesis by pharmacological or genetic inhibition of eNOS activity downregulates expression of the HIFs protein and concurrently abrogates the constitutively hypoxic phenotype of G1 cells (Figure 9E).
Overall, the molecular data reported here assign a master role to eNOS in the coordinated activation of a transcriptional program in response to estrogen and hypoxia. In this resides the innovative concept of eNOS as an essential cofactor of ER $\beta$ and HIFs in transcriptional regulation. The evidence of dynamic assembly on chromatin of functional combinatorial complexes among ER $\beta$ 

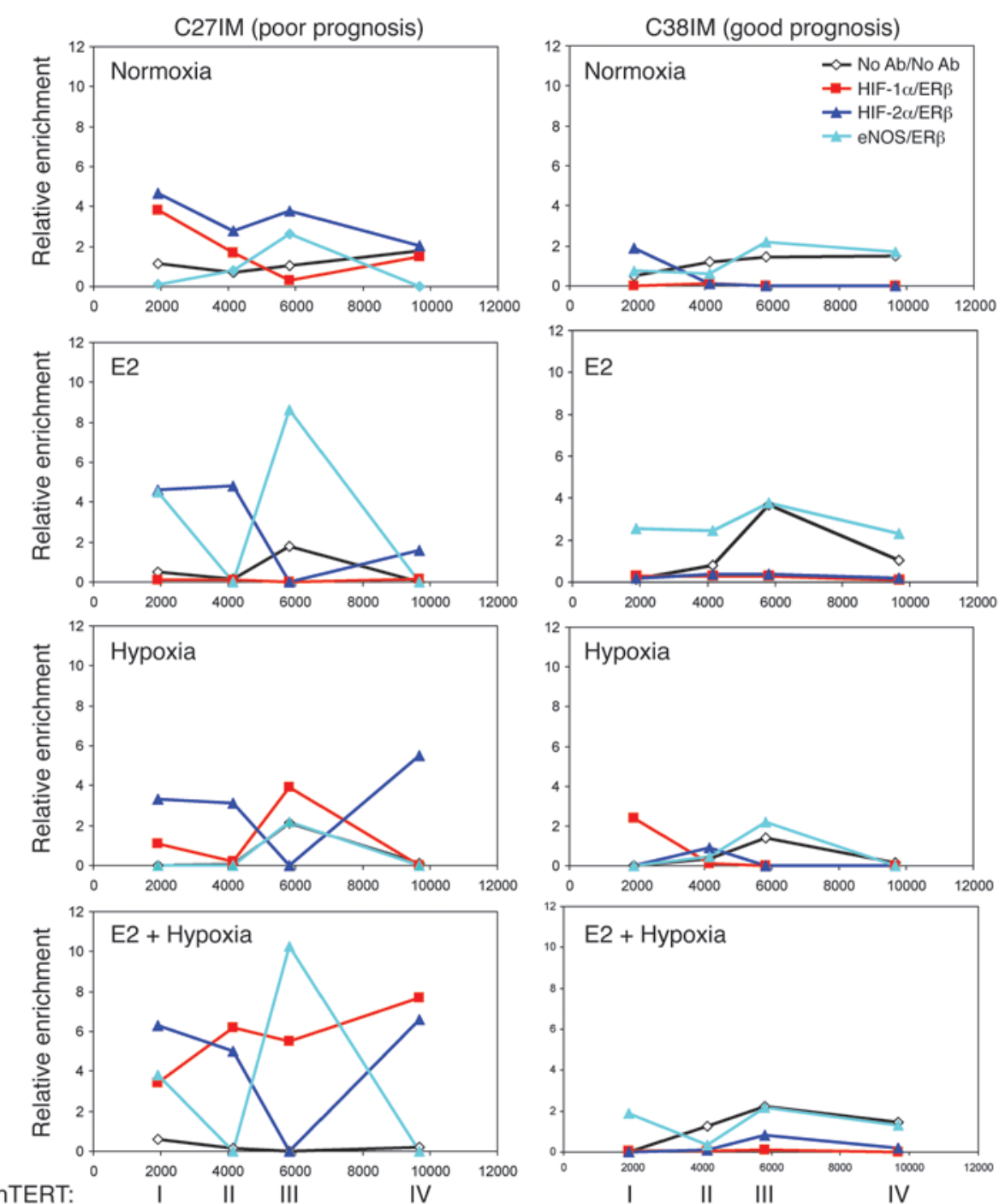

\section{Figure 8}

Analysis of the hTERT promoter by serial ChIP. Serial ChIP experiments were performed in parallel using C27IM and C38IM cells treated as in Figure 7. Immunoprecipitations were performed using antibodies to HIF- $1 \alpha$, HIF- $2 \alpha$, and eNOS or no antibody. Serial ChIPs were performed using antibodies to ER $\beta$ or no antibody. Co-recruitments of each transcription factor onto the hTERT promoter were detected by quantitative RT-PCR using primers specific for regions I-IV. The data represent the mean of 2 independent experiments. and eNOS or HIFs, highly sensitive to local changes in the hormonal and oxygen tension levels, substantially deepens our understanding of the molecular mechanisms underlying aggressiveness in hormone-dependent tumors. In particular, NO production and the constitutive phosphorylation and nuclear localization of eNOS appear to mark tumor cells more prone to progression. Consistent with this model, we recently demonstrated in endothelial cells that activated eNOS can translocate into the nucleus, in which it regulates gene transcription (18). The in vivo detection of association between nuclear eNOS and enhanced expression of HIF- $2 \alpha$ reinforces this concept and is in agreement with the observation that HIF- $2 \alpha$ seems to preferentially promote cell de-differentiation and proliferation (42). In addition, our accurate and systematic assessment of the prevalence and localization of eNOS within the cancer tissue contributes to the deciphering of the role of NO in tumor progression by overcoming the lack of such in vivo data.

eNOS is an important downstream target of the PI3K/AKT signaling pathway, which is strongly deregulated in our experimental system. In fact, in the original PCa primary cell populations, we found an extremely elevated expression of the serine/threonine kinase $\mathrm{AKT} /$ protein kinase $\mathrm{B}(A K T 1)$ mRNA by microarray as compared to the normal/hyperplastic cells (2). More interestingly, mRNA levels of $A K T 1$, a key regulator of cell survival, were significantly higher in $\mathrm{G} 1$ than in G2 cells $(P<0.05$; data not shown) and the AKT1 protein was constitutively phosphorylated (Figure 9E), reiterating the phenotypic difference of cells derived from patients with adverse prognosis. In line with this conclusion is our finding of the abrogation of hTERT-driven luciferase activity, basal and induced, upon pharmacological inhibition of the PI3K/AKT (Supplemental Figure 3), underscoring the requirement for AKT-mediated phosphorylation of eNOS in the regulation of hTERT promoter activity.

Resistance to apoptosis is another feature linked to the activation of PI3K/AKT and greatly enhanced by eNOS function (43), suggesting that reduced susceptibility to cell death may represent an important element toward acquisition of a more aggressive PCa phenotype. Although E2 appears to protect both G1 and G2 cells from apoptosis (data not shown), treatment with the testosterone metabolite $5 \alpha$-androstane- $3 \beta, 17 \beta$-diol, a specific ER $\beta$ ligand (44), prevented apoptosis in G1 cells while enhancing it in G2 cells. This suggests that this metabolite may play agonist or antagonist roles in different cell environments, substantiating our hypothesis of a 


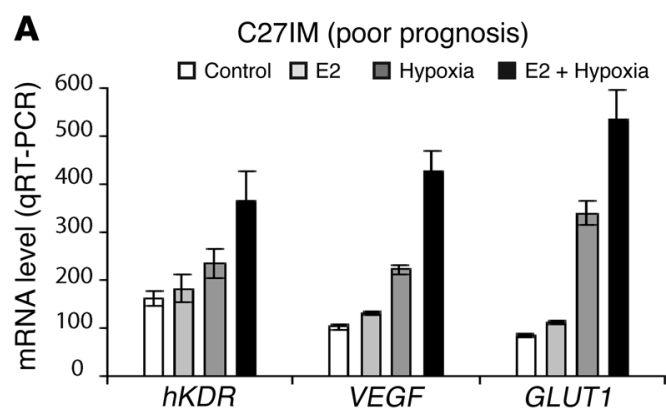

B C38IM (good prognosis)

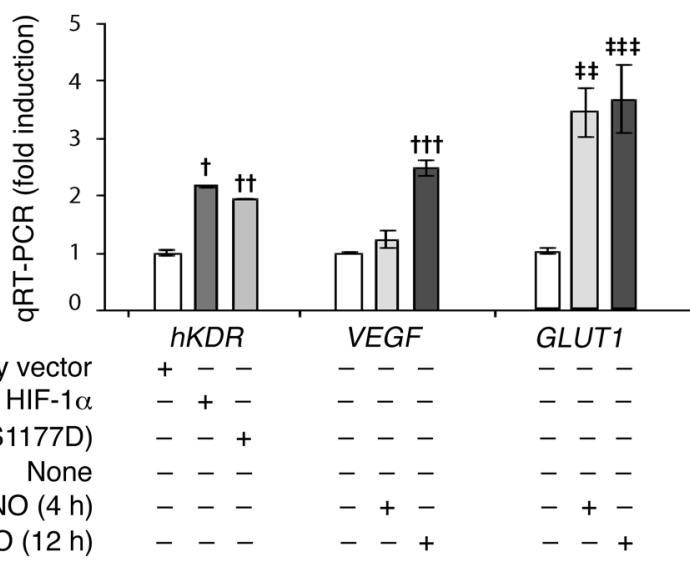

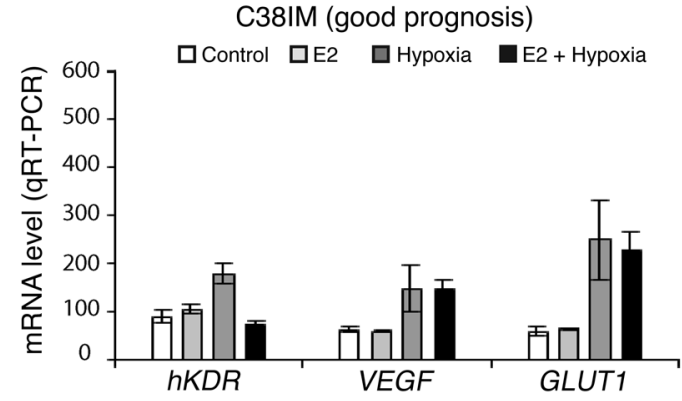

C

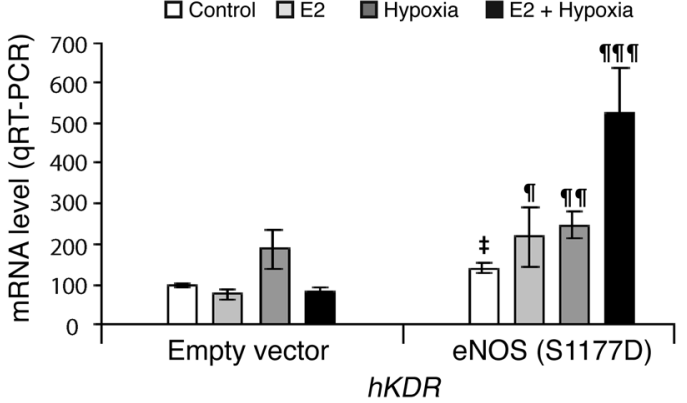

E
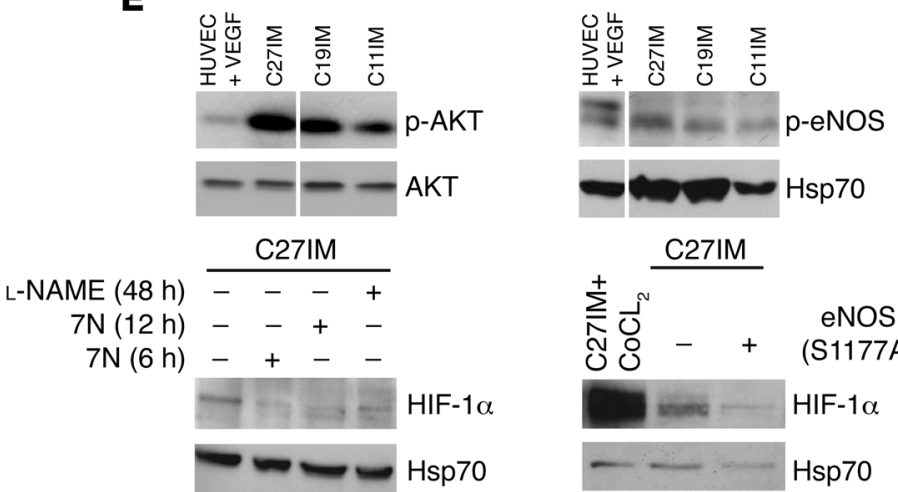

L-NAME $(48 \mathrm{~h}) \frac{786-0}{-} \frac{\mathrm{C} 27 \mathrm{IM}}{--+}$

$7 \mathrm{~N}(6 \mathrm{~h})$

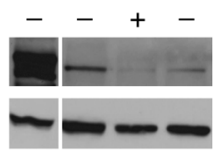

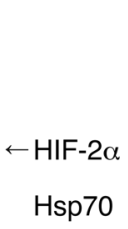

Figure 9

Expression of estradiol and/or hypoxia target genes and modulation of the transcriptional profile of PCa cells. (A) VEGF, hKDR, and GLUT1 mRNA levels were assessed by quantitative RT-PCR in C27IM (G1) and C38IM (G2) cells after 6 hours treatment with E2 (10-7 M), hypoxia $\left(1 \% \mathrm{O}_{2}\right)$, or both. Data represent mean \pm SEM of 3 experiments. (B and C) C38IM cells transfected with constitutively active HIF-1 $\alpha$ or eNOS in basal conditions, treated with DETA-NO for 4 and 12 hours, or under E2, hypoxia, or both. Data, plotted as fold induction in B, represent mean \pm SEM of 3 experiments. $(B){ }^{\dagger} P=0.0003,{ }^{\dagger} P=0.003$ versus empty vector; ${ }^{\dagger+t} P=0.0013, \neq \neq P<10^{-4}$, $\neq \pm P=0.0006$ versus basal condition.

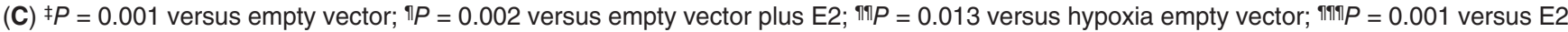
plus hypoxia empty vector. (D) G1 cells (C27IM and C19IM) treated with the NOS inhibitors 7-nitroindazole (7N) or N(G)-nitro-L-arginine methyl ester (L-NAME). Data, plotted as percentage, represent mean \pm SEM of 2 experiments. ${ }^{*} P<10^{-4}$ and ${ }^{* *} P<10^{-4}$ versus none for C27IM, ${ }^{\circ} P=0.01$ and ${ }^{\circ 0} P<10^{-4}$ versus none for $C 19 \mathrm{IM} ; \wedge P<10^{-4}$ and $M P<10^{-4}$ versus none for $\mathrm{C} 27 \mathrm{IM} ; \S P<10^{-4}$ and $\$ \S P=0.04$ versus none for $C 19 \mathrm{IM}$; $\# P<10^{-4}$ and ${ }^{\# \#} P<10^{-4}$ versus none for $\mathrm{C} 27 \mathrm{IM} ;{ }^{\sharp} P=0.05$ and ${ }^{\sharp} P=0.01$ versus none for C19IM. (E) Western blot of AKT, phosphorylated AKT, and eNOS in $\mathrm{G} 1$ cells (C27IM, C19IM, and C11IM) in basal condition and of HIF-1 $\alpha$ and HIF-2 $\alpha$ in C27IM treated with N(G)-nitro-L-arginine methyl ester or 7-nitroindazole or transfected with dominant-negative eNOS (S1177A). Hsp70 was the loading control.

deregulated estrogen signaling, exclusively in cells from tumors with more aggressive phenotype and resistance to apoptosis (2). This conclusion is also supported by the observations that the classical estro- gen target gene PS2 is overexpressed in G1 cells (Figure 1D), and that $\mathrm{ER} \beta$ and eNOS are both recruited to the PS2 promoter in response to estrogen and hypoxia stimuli (Supplemental Figure 1). 
Altogether, these data lead us to postulate that activation of eNOS is a crucial requirement for tumor progression in the prostate microenvironment, which is highly sensitive to abnormal estrogen level and hypoxia. ERs and the hypoxia-response family members are already exploited in clinical oncology as therapeutic targets in endocrine cancers. We contribute to this approach by adding a key molecule, eNOS, which together with abnormal ER and hypoxiaresponse signaling, drives $\mathrm{PCa}$ toward a more aggressive phenotype. These findings facilitate optimization of patient stratification for predicting clinical outcome and foster innovative therapies aimed at targeting the combined NO, estrogen, and hypoxia responses.

\section{Methods}

Generation of immortalized cell lines. Immortalized PCa cells were derived from primary cell cultures obtained from fresh tissue explants as described previously (2). Informed consent forms were signed by patients prior to surgery and experiments were approved by the Ethical Committee of the Regina Elena Cancer Institute. The cDNAs for DTERT and SV40 large T antigen were cloned into the retrovirus vectors PLXSP (45) and pBabepuro, respectively, both of which carry the puromycin resistance gene. Production of the retroviruses pLhTERTSP and pBabepuroTwt and infection of target cells were as described previously (45).

TMA construction, immunohistochemistry, and scoring. Paraffin-embedded tissues from 88 patients that had undergone prostatectomy for clinically localized PCa at the Urology Division, Regina Elena Cancer Institute, between 1990 and 1998, were used for TMA construction. None of the cancer patients had received neoadjuvant hormonal treatment or radiotherapy before surgery. The use of archival formalin-fixed, paraffinembedded prostate tumors from the Department of Pathology human tissue repository was approved by the Ethical Committee of the Regina Elena Cancer Institute.

The characteristics of the study group are detailed in Supplemental Table 1. The tissue blocks were selected, sectioned, and stained with H\&E and then graded (all histopathological data were systematically reevaluated by the pathologist) in accordance with the Union Internationale Contre le Cancer TNM system (UICC) (TNM Classification of Malignant Tumors. 6th edition. Wiley-Liss. 2002), prior to TMA construction. Representative areas of PCa were marked on both slide and matching paraffin tissue block for TMA construction. TMAs were prepared at the Medical Oncology Department of the Dana-Farber Cancer Institute, Harvard Medical School, Boston, USA. Briefly, 1 tissue cylinder with a diameter of $0.6 \mathrm{~mm}$ was punched from morphologically representative tissue areas of each "donor" tissue block and brought into 1 recipient paraffin block, using a homemade tissue arrayer. Two or three 0.6-mm diameter tissue cores (from tumoral and normal area) were taken from each PCa case. Four composite high-density TMA blocks were designed, and serial $5-\mu \mathrm{m}$ sections were cut with a Leica microtome and transferred to adhesive-coated slides. Immunohistochemistry was performed as described (2). The nuclear and cytoplasmic compartments were scored separately for all markers except for HIF- $1 \alpha$, which was mostly cytoplasmic. TMA sections were scored semiquantitatively based upon the proportion of tumor cells stained (quantity $[Q]$ ) and the staining intensity $(I)$ to obtain the final score as the product of $I \times Q(46,47)$. The scoring system was as follows for Q: 0 , negative; $1,1 \%-9 \%$ positive cells; $2,10 \%-39 \%$ positive cells; $3,40 \%-69 \%$ positive cells; $4,70 \%-100 \%$ positive cells. The scoring system was as follows for I: 0 , negative; 1 , low; 2 , moderate; 3 , high. Immunostaining was assessed by a histopathologist and reviewed independently by a second histopathologist, both blinded to patients outcome. Only representative tissue cores containing at least $20 \%$ of tumor cells were scored.

Outcome analysis. The outcome for the patients (survival, recurrence rate, biochemical recurrence) was followed up to June 2008 (observation period, January 1990 to June 2008). A complete database with all clinical and histopathological information was generated from the medical records of the Regina Elena Cancer Institute. Patients with PCa were divided in 2 groups based on clinical outcome: unfavorable prognosis was defined by presence of biochemical/local recurrence, metastasis, or disease-specific mortality, and favorable prognosis was defined by complete remission with surgery alone. The cause of death was classified as PCa, other disease, or undefined cause. The survival average was 12.9 years after surgery ( $95 \%$ confidence interval, $12.0-13.8$ years) and the median observation period was 9.9 years (25th-75th percentile, 6.2-11.2 years). Biochemical recurrence was defined according to the American Society for Therapeutic Radiology and Oncology (ASTRO) consensus definition (3 consecutive rises), with time of recurrence backdated to the midpoint between the PSA nadir and the first PSA rise (48), and the Phoenix definition (PSA nadir $+2 \mathrm{ng} / \mathrm{ml}$ ) (49).

Statistics. Statistical analysis was performed using Prism 2.01 statistical software (GraphPad). Differences among subject groups were assessed by 2-tailed Mann-Whitney $U$ test. A 95\% confidence interval $(P<0.05)$ was considered significant.

For TMA data analysis, the DSS, local or distant relapse-free survival, or biochemical recurrence rates were calculated using the Kaplan-Meier method from the time of prostatectomy (50). The statistical significance of differences in survival rates between groups was established by the logrank test. Hazards ratios and $95 \%$ confidence intervals were calculated for each biomarker as predictors of different end points in the Kaplan-Meier analysis. Multivariate analysis of prognostic factors was performed using the Cox proportional hazard model (51). The data analysis was performed with an SPSS version 10 (SPSS Inc.).

\section{Acknowledgments}

We thank F. Graham and S. Soddu for critical reading of the manuscript, D. Del Bufalo for reagents and helpful suggestions, and all members of the Division of Radiotherapy, Regina Elena Cancer Institute for constructive discussions. This work was supported by research grants from Associazione Italiana Ricerca sul Cancro and Ministero del Lavoro, della Salute e delle Politiche Sociali (to A. Farsetti); Ministero dell'Istruzione, dell'Università e della Ricerca; grants from NIH (Specialized Programs of Research Excellence 5P50CA90381, 5R01CA131945) and the Prostate Cancer Foundation (to M. Loda).

Received for publication January 18, 2008, and accepted in revised form February 11, 2009.

Address correspondence to: Antonella Farsetti, Department of Experimental Oncology, Molecular Oncogenesis Laboratory, Regina Elena Cancer Institute - Experimental Research Center (CRS), Via delle Messi d'Oro 156, 00158 Rome, Italy. Phone: 39-06-52662531; Fax: 39-06-41805-26; E-mail: afarsetti@gmail.com.
1. Ferlay, J., Bray, F., Pisani, P., and Parkin, D.M. 2004 GLOBOCAN 2002: Cancer incidence, mortality and prevalence worldwide. IARC CancerBase No. 5, version 2.0. IARC Press. Lyon, France. http://wwwdep.iarc.fr/.

2. Nanni, S., et al. 2006. Epithelial-restricted gene profile of primary cultures from human prostate tumors: a molecular approach to predict clinical behavior of prostate cancer. Mol. Cancer Res. 4:79-92.

3. Chi, J.T., et al. 2006. Gene expression programs in response to hypoxia: cell type specificity and prog- nostic significance in human cancers. PLoS Med. 3:e47.

4. Pouyssegur, J., Dayan, F., and Mazure, N.M. 2006. Hypoxia signalling in cancer and approaches to enforce tumour regression. Nature. 441:437-443.

5. Zhong, H., et al. 1999. Overexpression of hypoxia- 
inducible factor 1alpha in common human cancers and their metastases. Cancer Res. 59:5830-5835.

6. Dales, J.P., et al. 2005. Overexpression of hypoxiainducible factor HIF-1alpha predicts early relapse in breast cancer: retrospective study in a series of 745 patients. Int. J. Cancer. 116:734-739.

7. Gatenby, R.A., and Gillies, R.J. 2004. Why do cancers have high aerobic glycolysis? Nat. Rev. Cancer. 4:891-899.

8. Keith, B., and Simon, M.C. 2007. Hypoxia-inducible factors, stem cells, and cancer. Cell. 129:465-472.

9. Ho, S.M., Leung, Y.K., and Chung, I. 2006. Estrogens and antiestrogens as etiological factors and therapeutics for prostate cancer. Ann. N. Y. Acad. Sci. 1089:177-193.

10. Adams, J.Y., Leav, I., Lau, K.M., Ho, S.M., and Pflueger, S.M. 2002. Expression of estrogen receptor beta in the fetal, neonatal, and prepubertal human prostate. Prostate. 52:69-81.

11. Shapiro, E., et al. 2005. Immunolocalization of estrogen receptor alpha and beta in human fetal prostate. J. Urol. 174:2051-2053.

12. Signoretti, S., and Loda, M. 2001. Estrogen receptor beta in prostate cancer: brake pedal or accelerator? Am. J. Pathol. 159:13-16.

13. Imamov, O., Shim, G.J., Warner, M., and Gustafsson, J.A. 2005. Estrogen receptor beta in health and disease. Biol. Reprod. 73:866-871.

14. Zhu, X., et al. 2004. Dynamic regulation of estrogen receptor-beta expression by DNA methylation during prostate cancer development and metastasis. Am. J. Pathol. 164:2003-2012.

15. Horvath, L.G., et al. 2001. Frequent loss of estrogen receptor-beta expression in prostate cancer. Cancer Res. 61:5331-5335.

16. Leav, I., et al. 2001. Comparative studies of the estrogen receptors beta and alpha and the androgen receptor in normal human prostate glands, dysplasia, and in primary and metastatic carcinoma. Am. J. Pathol. 159:79-92.

17. Coulet, F., Nadaud, S., Agrapart, M., and Soubrier, F. 2003. Identification of hypoxia-response element in the human endothelial nitric-oxide synthase gene promoter. J. Biol. Chem. 278:46230-46240.

18. Grasselli, A., et al. 2008. Estrogen receptor-alpha and endothelial nitric oxide synthase nuclear complex regulates transcription of human telomerase. Circ. Res. 103:34-42.

19. Zeng, L., and Xu, Q. 2008. eNOS-ERalpha complex goes to telomerase. Circ. Res. 103:10-12.

20. Chambliss, K.L., and Shaul, P.W. 2002. Estrogen modulation of endothelial nitric oxide synthase. Endocr. Rev. 23:665-686.

21. Brune, B., and Zhou, J. 2007. Nitric oxide and superoxide: interference with hypoxic signaling. Cardiovasc. Res. 75:275-282.

22. Sandau, K.B., Fandrey, J., and Brune, B. 2001. Accumulation of HIF-1alpha under the influence of nitric oxide. Blood. 97:1009-1015.

23. Kasuno, K., et al. 2004. Nitric oxide induces hypoxia-inducible factor 1 activation that is dependent on MAPK and phosphatidylinositol 3-kinase signaling. J. Biol. Chem. 279:2550-2558.

24. Thomas, D.D., et al. 2004. Hypoxic inducible factor 1alpha, extracellular signal-regulated kinase, and p53 are regulated by distinct threshold concentrations of nitric oxide. Proc. Natl. Acad. Sci. U. S. A 101:8894-8899.

25. Lim, K.H., Ancrile, B.B., Kashatus, D.F., and Counter, C.M. 2008. Tumour maintenance is mediated by eNOS. Nature. 452:646-649.

26. Fukumura, D., Kashiwagi, S., and Jain, R.K. 2006 The role of nitric oxide in tumour progression. Nat. Rev. Cancer. 6:521-534.

27. Ying, L., and Hofseth, L.J. 2007. An emerging role for endothelial nitric oxide synthase in chronic inflammation and cancer. Cancer Res. 67:1407-1410.

28. Zhang, W., Kapusta, L.R., Slingerland, J.M., and Klotz, L.H. 1998. Telomerase activity in prostate cancer, prostatic intraepithelial neoplasia, and benign prostatic epithelium. Cancer Res. 58:619-621.

29. Sommerfeld, H.J., et al. 1996. Telomerase activity: a prevalent marker of malignant human prostate tissue. Cancer Res. 56:218-222.

30. Semenza, G.L. 2003. Targeting HIF-1 for cancer therapy. Nat. Rev. Cancer. 3:721-732.

31. Kyo, S., et al. 1999. Estrogen activates telomerase. Cancer Res. 59:5917-5921.

32. Nishi, H., et al. 2004. Hypoxia-inducible factor 1 mediates upregulation of telomerase (hTERT). Mol. Cell. Biol. 24:6076-6083.

33. Carroll, J.S., et al. 2005. Chromosome-wide mapping of estrogen receptor binding reveals longrange regulation requiring the forkhead protein FoxA1. Cell. 122:33-43.

34. Nanni, S., et al. 2002. Signaling through estrogen receptors modulates telomerase activity in human prostate cancer. J. Clin. Invest. 110:219-227.

35. Shang, Y., Hu, X., DiRenzo, J., Lazar, M.A., and Brown, M. 2000. Cofactor dynamics and sufficiency in estrogen receptor-regulated transcription. Cell. 103:843-852.

36. Jiang, B.H., Semenza, G.L., Bauer, C., and Marti, H.H. 1996. Hypoxia-inducible factor 1 levels vary exponentially over a physiologically relevant range of $\mathrm{O} 2$ tension. Am. J. Physiol. 271:C1172-C1180.

37. Seifeddine, R., et al. 2007. Hypoxia and estrogen co-operate to regulate gene expression in T-47D human breast cancer cells. J. Steroid Biochem. Mol. Biol. 104:169-179.

38. Venkov, C.D., Rankin, A.B., and Vaughan, D.E. 1996. Identification of authentic estrogen receptor in cultured endothelial cells. A potential mechanism for steroid hormone regulation of endothelial function. Circulation. 94:727-733.

39. Klinge, C.M. 1999. Role of estrogen receptor ligand and estrogen response element sequence on interaction with chicken ovalbumin upstream promoter transcription factor (COUP-TF). J. Steroid Biochem. Mol. Biol. 71:1-19.

40. Krishnamachary, B., and Semenza, G.L. 2007. Analysis of hypoxia-inducible factor 1alpha expression and its effects on invasion and metastasis. Methods Enzymol. 435:347-354.

41. Kimbro, K.S., and Simons, J.W. 2006. Hypoxiainducible factor- 1 in human breast and prostate cancer. Endocr. Relat. Cancer. 13:739-749.

42. Gordan, J.D., and Simon, M.C. 2007. Hypoxiainducible factors: central regulators of the tumor phenotype. Curr. Opin. Genet. Dev. 17:71-77.

43. Mattiussi, S., et al. 2006. Papilloma protein E6 abrogates shear stress-dependent survival in human endothelial cells: evidence for specialized functions of paxillin. Cardiovasc. Res. 70:578-588.

44. Weihua, Z., etal. 2001. A role for estrogen receptor beta in the regulation of growth of the ventral prostate. Proc. Natl. Acad. Sci. U. S. A. 98:6330-6335.

45. Bossi, G., et al. 2004. Wild-type p53 gene transfer is not detrimental to normal cells in vivo: implications for tumor gene therapy. Oncogene. 23:418-425.

46. Signoretti, S., et al. 2002. Oncogenic role of the ubiquitin ligase subunit Skp2 in human breast cancer. J. Clin. Invest. 110:633-641.

47. Ogino, S., et al. 2008. Cohort study of fatty acid synthase expression and patient survival in colon cancer. J. Clin. Oncol. 26:5713-5720.

48. [No authors listed]. 1997. Consensus statement: guidelines for PSA following radiation therapy. American Society for Therapeutic Radiology and Oncology Consensus Panel. Int. J. Radiat. Oncol. Biol. Phys. 37:1035-1041.

49. Roach, M., 3rd, et al. 2006. Defining biochemical failure following radiotherapy with or without hormonal therapy in men with clinically localized prostate cancer: recommendations of the RTOGASTRO Phoenix Consensus Conference. Int. J. Radiat. Oncol. Biol. Phys. 65:965-974.

50. Kaplan, E.L., and Meier, P. 1958. Nonparametric estimation from incomplete observations. J. Am. Stat. Assoc. 53:457-481.

51. Cox, D.R. 1975. Partial likelihood. Biometrika. 62:269-276. 